\title{
To be published
}

\section{Domino 'Staudinger / Semi-Aza-Wittig / Fragmentation' Reactions of $\gamma$-Azido- $\beta$-hydroxyketones}

\author{
Ilia Freifeld ${ }^{a, b}$ Heydar Shojaei, ${ }^{b}$ Rüdiger Dede, ${ }^{c}$ and Peter Langer* ${ }^{c, d}$ \\ ${ }^{a}$ Institut für Biochemie, Universität Greifswald, \\ Soldmannstr. 16, 17487 Greifswald, Germany \\ ${ }^{\mathrm{b}}$ Institut für Organische und Biomolekulare Chemie, Georg-August-Universität Göttingen, \\ Tammannstr. 2, 37077 Göttingen, Germany \\ ${ }^{\mathrm{c}}$ Institut für Chemie, Universität Rostock, \\ Albert-Einstein-Str. 3a, 18059 Rostock, Germany \\ Fax:+381 4986412,E-mail: peter.langer@uni-rostock.de \\ ${ }^{\mathrm{d}}$ Leibniz-Institut für Katalyse e. V. an der Universität Rostock, \\ Albert-Einstein-Str. 29a, 18059 Rostock, Germany
}

\section{Supplementary Information}

\section{Contents:}


General. All solvents were dried by standard methods and all reactions were carried out under an inert atmosphere. For ${ }^{1} \mathrm{H}$ and ${ }^{13} \mathrm{C}$ NMR spectra the deuterated solvents indicated were used. Mass spectrometric data (MS) were obtained by electron ionization (EI, $70 \mathrm{eV})$, chemical ionization $\left(\mathrm{CI}, \mathrm{H}_{2} \mathrm{O}\right)$ or electrospray ionization (ESI). For preparative scale chromatography, silica gel (60-200 mesh) was used. Melting points are uncorrected. The $\alpha-$ azidoketones 2a-f were prepared according to literature procedures. 2-Azidocyclopentanone (2f) was prepared from 2-chlorocyclopentanone. ${ }^{1}$ 2-Azidoindan-1-one (2b) was prepared in 3 steps by a literature procedure. ${ }^{2}$ 1-Azidopropan-2-one (2c) was prepared from 3-chlorobutan2-one and $\mathrm{NaN}_{3}{ }^{3}{ }^{3}$ 3-Azidobutan-2-one (2d) was prepared by reaction of 3-chlorobutan-2-one with $\mathrm{NaN}_{3}{ }^{4}$ 2-Azidocyclohexanone (2e) was prepared from 2-chlorocyclohexanone. ${ }^{5}$

CAUTION: The handling of azides is dangerous, due to their potentially explosive character. Although, in our hands, azides 2 did not appear to be shock sensitive, the compounds should be handled with great care. Neat azides must not be heated or distilled and all reactions should be carried out on a small scale. The use of a safety shield is highly recommended.

\section{General procedure for the synthesis of 2-azido-1-hydroxy-1-(2,4-} dioxoalkyl)cyclopentanes 3a-w: To a solution of diisopropylamine (2.6 equiv) in anhydrous THF (35 ml) was added $n \operatorname{BuLi}\left(2.6\right.$ equiv, $23 \%$ or $15 \%$ solution in hexanes) at $0{ }^{\circ} \mathrm{C}$. After stirring for 15 min the dicarbonyl compound 1 (1.1 equiv) was added and the solution was stirred for $1 \mathrm{~h}$ at $0{ }^{\circ} \mathrm{C}$. A THF solution $(5 \mathrm{ml})$ of azidoketone 2 (1.0 equiv) was added at -78 ${ }^{\circ} \mathrm{C}$ and the reaction mixture was warmed to ambient temperature during $12 \mathrm{~h}$. After stirring for further $3 \mathrm{~h}$ at $20^{\circ} \mathrm{C}$ a saturated aqueous solution of $\mathrm{NH}_{4} \mathrm{Cl}(50 \mathrm{ml})$ was added, the organic 
layer was separated and the aqueous layer was extracted with $\mathrm{Et}_{2} \mathrm{O}(2 \times 70 \mathrm{ml})$ and with $\mathrm{CH}_{2} \mathrm{Cl}_{2}(2 \times 50 \mathrm{ml})$. The combined organic layers were extracted with brine, dried $\left(\mathrm{Na}_{2} \mathrm{SO}_{4}\right)$, filtered and the solvent of the filtrate was removed in vacuo. The residue was purified by chromatography (silica gel, ether/petroleum ether $=1: 4 \rightarrow 1: 3$ ) to give the alcohol 3 . Due to its instability, the product should be used for further transformations within $24 \mathrm{~h}$. In some cases, small amounts of decomposition products could not be removed. The following products reside in their enolic form: 3n, 3o, 3p, 3q, 3r, 3s, 3t, 3u, 3w, 3x, 3y.

Ethyl 4-(2-azido-1-hydroxycyclopentyl)-3-oxobutyrate (3a): The starting materials diisopropylamine (0.63 g, $6.30 \mathrm{mmol}), n \mathrm{BuLi}(4.1 \mathrm{ml}, 6.30 \mathrm{mmol}, 15 \%$ solution in hexane), ethyl acetoacetate (1a) $(0.34 \mathrm{~g}, 2.64 \mathrm{mmol})$ and 2-azidocyclopentanone (2a) $\quad(0.30 \mathrm{~g}, 2.40$ mmol) yielded 3a as a yellow oil (0.51 g, 57\%, dr > 98:2). ${ }^{1} \mathrm{H}-\mathrm{NMR}\left(300 \mathrm{MHz}, \mathrm{CDCl}_{3}\right.$, major isomer): $\delta=1.25\left(\mathrm{t},{ }^{3} J=7 \mathrm{~Hz}, 3 \mathrm{H}\right), 1.55-2.04(\mathrm{~m}, 6 \mathrm{H}), 2.63\left(\mathrm{~d},{ }^{2} J=16 \mathrm{~Hz}, 1 \mathrm{H}\right)$, $2.98\left(\mathrm{~d},{ }^{2} J=16 \mathrm{~Hz}, 1 \mathrm{H}\right), 3.32\left(\mathrm{t},{ }^{3} \mathrm{~J}=6 \mathrm{~Hz}, 1 \mathrm{H}\right), 3.45(\mathrm{~s}, 2 \mathrm{H}), 4.18\left(\mathrm{q},{ }^{3} J=7 \mathrm{~Hz}, 2 \mathrm{H}\right) .{ }^{13} \mathrm{C}-$ $\operatorname{NMR}\left(50.3 \mathrm{MHz}, \mathrm{CDCl}_{3}\right.$, major isomer): $\delta=14.0,19.2,26.9,36.0,49.8,50.4,61.5,67.7$, 79.8, 166.9, 203.3. IR (neat): $\tilde{v}=3503$ (br, w), 2978 (w), 2106 (d), 1740 (s), 1711 (s), 1446 (w), 1407 (w), 1369 (w), $1318(\mathrm{~m}), 1258(\mathrm{~m}), 1181(\mathrm{~m}), 1096(\mathrm{w}), 1028(\mathrm{~m}) \mathrm{cm}^{-1}$. MS (DCI, $\left.\mathrm{NH}_{3}\right): m / z(\%)=528\left(\left[2 \mathrm{M}+\mathrm{NH}_{4}\right]^{+}, 84\right), 483(80), 438(22), 273\left(\left[\mathrm{M}+\mathrm{NH}_{4}\right]^{+}, 100\right), 228(36)$, 210 (18). Anal. calcd for $\mathrm{C}_{11} \mathrm{H}_{17} \mathrm{~N}_{3} \mathrm{O}_{4}$ (255.27): C 51.70, H 6.66; found: C 51.59, H 6.43.

Methyl 4-(2-azido-1-hydroxycyclopentyl)-3-oxobutyrate (3b): The starting materials diisopropylamine $(0.63 \mathrm{~g}, 6.30 \mathrm{mmol}), n \mathrm{BuLi}(4.1 \mathrm{ml}, 6.30 \mathrm{mmol}, 15 \%$ solution in hexane), methyl acetoacetate (1b) (0.31 g, $2.64 \mathrm{mmol})$ and 2-azidocyclopentanone (2a) $(0.30 \mathrm{~g}, 2.40$ mmol) yielded $\mathbf{3 b}$ as a yellow oil $\left(0.32 \mathrm{~g}, 55 \%\right.$, dr > 98:2). ${ }^{1} \mathrm{H}-\mathrm{NMR}\left(250 \mathrm{MHz}, \mathrm{CDCl}_{3}\right.$, major isomer): $\delta=1.50-2.05(\mathrm{~m}, 6 \mathrm{H}), 2.65\left(\mathrm{~d},{ }^{2} J=17 \mathrm{~Hz}, 1 \mathrm{H}\right), 2.98\left(\mathrm{~d},{ }^{2} J=17 \mathrm{~Hz}, 1 \mathrm{H}\right)$, 
$3.32\left(\mathrm{t},{ }^{3} \mathrm{~J}=7 \mathrm{~Hz}, 1 \mathrm{H}\right), 3.52(\mathrm{~s}, 2 \mathrm{H}), 3.73(\mathrm{~s}, 3 \mathrm{H}) .{ }^{13} \mathrm{C}-\mathrm{NMR}\left(50.3 \mathrm{MHz}, \mathrm{CDCl}_{3}\right.$, major isomer): $\delta=10.0,26.9,35.9,49.8,50.1,52.4,67.6,79.8,167.3,203.1$. IR (neat): $\tilde{v}=3502$ (w), 2956 (m), 2105 (s), 1744 (s), 1711 (s), 1654 (w), 1438 (m), 1258 (m), 1161 (m), 1100 (w), $1009(\mathrm{w}), 866(\mathrm{w}), 558(\mathrm{w}) \mathrm{cm}^{-1}$. MS (DCI, $\left.\mathrm{NH}_{3}\right): \mathrm{m} / z(\%)=500\left(\left[2 \mathrm{M}+\mathrm{NH}_{4}\right]^{+}, 76\right), 455$ (100), 410 (32), $259\left(\left[\mathrm{M}+\mathrm{NH}_{4}\right]^{+}, 81\right), 214$ (61). Anal. calcd for $\mathrm{C}_{10} \mathrm{H}_{15} \mathrm{~N}_{3} \mathrm{O}_{4}$ (241.25): C 49.70, H 6.22, N 17.41; found: C 49.87, H 6.05, N 17.46.

Benzyl 4-(2-azido-1-hydroxycyclopentyl)-3-oxobutyrate (3c): The starting materials diisopropylamine $(0.52 \mathrm{~g}, 5.20 \mathrm{mmol}), n \mathrm{BuLi}(2.2 \mathrm{ml}, 5.20 \mathrm{mmol}, 23 \%$ solution in hexanes $)$, benzyl acetoacetate (1c) $(0.42 \mathrm{~g}, 2.20 \mathrm{mmol})$ and 2-azidocyclopentanone (2a) $(0.25 \mathrm{~g}, 2.00$ mmol) yielded 3c as a yellow oil (0.21 g, 35\%, dr > 10:1). ${ }^{1} \mathrm{H} \mathrm{NMR}\left(300 \mathrm{MHz}, \mathrm{CDCl}_{3}\right.$, major isomer): $\delta=1.59(\mathrm{~m}, 2 \mathrm{H}), 2.94(\mathrm{~m}, 4 \mathrm{H}), 2.59\left(\mathrm{~d},{ }^{2} J=17 \mathrm{~Hz}, 1 \mathrm{H}\right), 2.95\left(\mathrm{~d},{ }^{2} J=17 \mathrm{~Hz}, 1\right.$ $\mathrm{H}), 3.27\left(\mathrm{t},{ }^{3} \mathrm{~J}=7 \mathrm{~Hz}, 1 \mathrm{H}\right), 3.54(\mathrm{~s}, 2 \mathrm{H}), 5.27(\mathrm{~s}, 2 \mathrm{H}), 7.35(\mathrm{~m}, 5 \mathrm{H}) .{ }^{13} \mathrm{C}$ NMR $(75.5 \mathrm{MHz}$, $\mathrm{CDCl}_{3}$, major isomer): $\delta=19.2,26.9,35.9,49.8,50.4,67.3,67.7,79.8,128.2,128.4,128.6$, 135.0, 166.7, 203.0. IR (neat): $\tilde{v}=3465$ (br, w), 2958 (m), 2930 (m), 2106 (s), 1741 (m), 1711 (m), 1646 (w), 1452 (m), 1406 (m), 1379 (m), 1317 (m), 1317 (m), 1261 (m), 1155 (m), 1098 (m), 1050 (w), 1025 (w), 1000 (w), 748 (w), 699 (w) $\mathrm{cm}^{-1}$. MS (DCI, NH $\left.\mathrm{NH}_{3}\right): \mathrm{m} / \mathrm{z}$ $(\%)=652\left(\left(2 \mathrm{M}+\mathrm{NH}_{4}\right)^{+}, 5\right), 335\left(\left(\mathrm{M}+\mathrm{NH}_{4}\right)^{+}, 100\right), 318\left((\mathrm{M}+\mathrm{H})^{+}, 23\right), 290(11), 272(5), 182$ (7), $133(8)$.

2-Methoxyethyl 4-(2-azido-1-hydroxycyclopentyl)-3-oxobutyrate (3d): The starting materials diisopropylamine $(0.52 \mathrm{~g}, 5.20 \mathrm{mmol}), n \mathrm{BuLi}(2.2 \mathrm{ml}, 5.20 \mathrm{mmol}, 23 \%$ solution in hexanes), 2-methoxyethyl acetoacetate (1d) $(0.35 \mathrm{~g}, 2.20 \mathrm{mmol})$ and 2-azidocyclopentanone (2a) $(0.25 \mathrm{~g}, 2.00 \mathrm{mmol})$ yielded 3d as a yellow oil $(0.37 \mathrm{~g}, 64 \%, \mathrm{dr}=10: 1) .{ }^{1} \mathrm{H}$ NMR (300 $\mathrm{MHz}, \mathrm{CDCl}_{3}$, major isomer): $\delta=1.62(\mathrm{~m}, 2 \mathrm{H}), 1.93(\mathrm{~m}, 4 \mathrm{H}), 2.62\left(\mathrm{~d},{ }^{2} J=17 \mathrm{~Hz}\right), 2.97(\mathrm{~d}$, 
$\left.{ }^{2} J=17 \mathrm{~Hz}, 1 \mathrm{H}\right), 3.31\left(\mathrm{t},{ }^{3} J=7 \mathrm{~Hz}, 1 \mathrm{H}\right), 3.34(\mathrm{~s}, 3 \mathrm{H}), 3.53(\mathrm{~s}, 2 \mathrm{H}), 3.57\left(\mathrm{t},{ }^{3} J=5 \mathrm{~Hz}, 2 \mathrm{H}\right)$, $4.26\left(\mathrm{t},{ }^{3} \mathrm{~J}=5 \mathrm{~Hz}, 2 \mathrm{H}\right) .{ }^{13} \mathrm{C} \mathrm{NMR}\left(75.5 \mathrm{MHz}, \mathrm{CDCl}_{3}\right.$, major isomer): $\delta=19.2,26.9,36.0$, 49.8, 50.3, 59.4, 64.2, 67.7, 70.0, 79.8, 166.9, 203.0. IR (neat): $\tilde{v}=3472$ (br, m), 2969 (m), 2954 (m), 2884 (m), 2105 (s), 1743 (s), 1712 (s), 1647 (m), 1449 (m), 1406 (m), 1372 (m), 1316 (m), 1258 (s), 1198 (m), 1158 (m), 1129 (m), 1100 (m), 1035 (m) cm ${ }^{-1}$. MS (DCI, $\left.\mathrm{NH}_{3}\right): m / z(\%)=588\left(\left(2 \mathrm{M}+\mathrm{NH}_{4}\right)^{+}, 1\right), 303\left(\left(\mathrm{M}+\mathrm{NH}_{4}\right)^{+}, 100\right), 286\left((\mathrm{M}+\mathrm{H})^{+}, 66\right), 258(12)$, $182(26)$.

Isoutyl 4-(2-azido-1-hydroxycyclopentyl)-3-oxo-butyrate (3e): The starting materials diisopropylamine $(0.52 \mathrm{~g}, 5.20 \mathrm{mmol}), n \mathrm{BuLi}(2.2 \mathrm{ml}, 5.20 \mathrm{mmol}, 23 \%$ solution in hexanes), isobutyl acetoacetate (1e) $(0.35 \mathrm{~g}, 2.20 \mathrm{mmol})$ and 2-azidocyclopentanone (2a) $(0.25 \mathrm{~g}, 2.00$ mmol) yielded 3e as a yellow oil $(0.46 \mathrm{~g}, 82 \%, \mathrm{dr}=10: 1) .{ }^{1} \mathrm{H} \mathrm{NMR}\left(300 \mathrm{MHz}, \mathrm{CDCl}_{3}\right.$, major isomer): $\delta=0.90\left(\mathrm{~d},{ }^{3} J=6 \mathrm{~Hz}, 6 \mathrm{H}\right), 1.61(\mathrm{~m}, 2 \mathrm{H}), 1.93(\mathrm{~m}, 5 \mathrm{H}), 2.63\left(\mathrm{~d},{ }^{2} J=17 \mathrm{~Hz}, 1 \mathrm{H}\right)$, $2.97\left(\mathrm{~d},{ }^{2} J=17 \mathrm{~Hz}, 1 \mathrm{H}\right), 3.32\left(\mathrm{t},{ }^{3} \mathrm{~J}=7 \mathrm{~Hz}, 1 \mathrm{H}\right), 3.50(\mathrm{~s}, 2 \mathrm{H}), 3.91\left(\mathrm{~d},{ }^{3} J=6 \mathrm{~Hz}, 2 \mathrm{H}\right) .{ }^{13} \mathrm{C}$ NMR (75.5 MHz, $\mathrm{CDCl}_{3}$, major isomer): $\delta=18.9,19.2,26.9,27.5,36.0,49.8,50.3,67.7$, 71.5, 79.8, 166.9, 203.3. IR (neat): $\tilde{v}=3501$ (br, w), 2964 (s), 2877 (m), 2106 (s), 1739 (s), $1711(\mathrm{~s}), 1649$ (w), 1468 (w), 1406 (m), 1376 (m), 1318 (m), 1258 (s), 1175 (m), 1157 (m), $1003(\mathrm{w}) \mathrm{cm}^{-1}$. MS (DCI, $\left.\mathrm{NH}_{3}\right): m / z(\%)=584\left(\left(2 \mathrm{M}+\mathrm{NH}_{4}\right)^{+}, 34\right), 301\left(\left(\mathrm{M}+\mathrm{NH}_{4}\right)^{+}, 100\right), 284$ $\left((\mathrm{M}+\mathrm{H})^{+}, 46\right), 256(20), 182(13)$.

Methyl 4-(2-azido-1-hydroxycyclopentyl)-4-methyl-3-oxovalerate (3f): The starting materials diisopropylamine $(1.05 \mathrm{~g}, 10.50 \mathrm{mmol}), n \mathrm{BuLi}(6.9 \mathrm{ml}, 10.05 \mathrm{mmol}, 15 \%$ solution in hexanes), methyl 3-oxovalerate (1f) $(0.57 \mathrm{~g}, 4.40 \mathrm{mmol})$ and 2-azidocyclopentanone (2a) $(0.50 \mathrm{~g}, 4.00 \mathrm{mmol})$ yielded $\mathbf{3 f}$ as a yellow oil $(0.34 \mathrm{~g}, 33 \%, 3$ stereocenters, mixture of 2 diastereomers). ${ }^{1} \mathrm{H}-\mathrm{NMR}$ (300 MHz, $\mathrm{CDCl}_{3}$, major isomer): $\delta=1.29\left(\mathrm{~d},{ }^{3} \mathrm{~J}=6 \mathrm{~Hz}, 3 \mathrm{H}\right), 1.45$ 
- $2.11(\mathrm{~m}, 6 \mathrm{H}), 2.78\left(\mathrm{q},{ }^{3} \mathrm{~J}=6 \mathrm{~Hz}, 1 \mathrm{H}\right), 3.45\left(\mathrm{t},{ }^{3} \mathrm{~J}=9 \mathrm{~Hz}, 1 \mathrm{H}\right), 3.52(\mathrm{~s}, 2 \mathrm{H}), 3.70(\mathrm{~s}, 3 \mathrm{H})$.

${ }^{13} \mathrm{C}-\mathrm{NMR}$ (50.3 MHz, $\mathrm{CDCl}_{3}$, major isomer): $\delta=12.0,19.5,28.4,35.4,48.8,51.0,52.4$, 66.3, 81.4, 167.5, 206.7. IR (neat) $\tilde{v}=3586(\mathrm{br}, \mathrm{w}), 2955(\mathrm{w}), 2106(\mathrm{~s}), 1745(\mathrm{~s}), 1708(\mathrm{~m})$, $1652(\mathrm{w}), 1629(\mathrm{w}), 1436(\mathrm{~m}), 1404(\mathrm{w}), 1319(\mathrm{~m}), 1257(\mathrm{~m}), 1181(\mathrm{w}), 1152(\mathrm{w}), 1079(\mathrm{w})$, $1003(\mathrm{w}) \mathrm{cm}^{-1}$. MS $\left(\mathrm{DCI}, \mathrm{NH}_{3}\right): \mathrm{m} / z(\%)=528\left(\left[2 \mathrm{M}+\mathrm{NH}_{4}\right]^{+}, 60\right), 483(30), 273\left(\left[\mathrm{M}+\mathrm{NH}_{4}\right]^{+}\right.$, 100), $256\left([\mathrm{M}+\mathrm{H}]^{+}, 6\right), 228$ (18). Anal. calcd for $\mathrm{C}_{11} \mathrm{H}_{17} \mathrm{~N}_{3} \mathrm{O}_{4}$ (255.27): C 51.70, H 6.66, N 16.45; found: C 51.87, H 6.48, N 16.18.

Ethyl 4-(2-azido-1-hydroxycyclopentyl)-3-oxohexanoate (3g): The starting materials diisopropylamine $(0.63 \mathrm{~g}, 6.30 \mathrm{mmol}), n \mathrm{BuLi}(4.1 \mathrm{ml}, 6.30 \mathrm{mmol}, 15 \%$ solution in hexanes), 3-oxohexanoate (1g) (0.42 g, $2.64 \mathrm{mmol})$ and 2-azidocyclopentanone (2a) (0.30 g, 2.40 $\mathrm{mmol})$ yielded $3 \mathrm{~g}$ as a yellow oil $(0.44 \mathrm{~g}, 65 \%, 3$ stereocenters, mixture of 2 diastereomers). ${ }^{1} \mathrm{H}-\mathrm{NMR}$ (250 MHz, $\mathrm{CDCl}_{3}$, major isomer): $\delta=0.88(\mathrm{~m}, 3 \mathrm{H}), 1.27(\mathrm{~m}, 3 \mathrm{H}), 1.22-2.10(\mathrm{~m}$, $8 \mathrm{H}), 2.76(\mathrm{~m}, 1 \mathrm{H}), 3.46(\mathrm{~m}, 2 \mathrm{H}), 3.58(\mathrm{~m}, 1 \mathrm{H}), 4.20\left(\mathrm{q},{ }^{3} \mathrm{~J}=7 \mathrm{~Hz}, 2 \mathrm{H}\right) .{ }^{13} \mathrm{C}-\mathrm{NMR}(50.3$ $\mathrm{MHz}, \mathrm{CDCl}_{3}$, major isomer): $\delta=12.2,14.0,19.0,21.9,26.8,33.9,52.8,60.2,66.5,83.0$, 92.4, 166.8, 207.8. MS (DCI, $\left.\mathrm{NH}_{3}\right): m / \mathrm{z}(\%)=584\left(\left[2 \mathrm{M}+\mathrm{NH}_{4}\right]^{+}, 4\right), 301\left(\left[\mathrm{M}+\mathrm{NH}_{4}\right]^{+}, 100\right)$, $284\left([\mathrm{M}+\mathrm{H}]^{+}, 9\right), 273$ (34), 256 (60). Anal. calcd for $\mathrm{C}_{13} \mathrm{H}_{21} \mathrm{~N}_{3} \mathrm{O}_{4}$ (283.33): C 55.11, H 7.44, N 14.82; found: C 55.06, H 7.58, N 14.67.

Methyl 4-(2-azido-1-hydroxycyclopentyl)-4-methoxy-3-oxobutyrate (3h): The starting materials diisopropylamine $(0.63 \mathrm{~g}, 6.30 \mathrm{mmol}), n \mathrm{BuLi}(4.1 \mathrm{ml}, 6.30 \mathrm{mmol}, 15 \%$ solution in hexane), methyl 4-methoxy-3-oxobutyrate (1h) $\quad\left(\begin{array}{lllll}0.39 & \mathrm{~g}, & 2.64 & \mathrm{mmol}\end{array}\right)$ and 2azidocyclopentanone (2a) $(0.30 \mathrm{~g}, 2.40 \mathrm{mmol})$ yielded $\mathbf{3 h}$ as a yellow oil $(0.35 \mathrm{~g}, 54 \%, 3$ stereocenters, mixture of 2 diastereomers). ${ }^{1} \mathrm{H}-\mathrm{NMR}$ (250 $\mathrm{MHz}, \mathrm{CDCl}_{3}$, major isomer): $\delta=$ $1.45-2.10(\mathrm{~m}, 6 \mathrm{H}), 3.34-3.52(\mathrm{~m}, 5 \mathrm{H}), 3.58-3.78(\mathrm{~m}, 5 \mathrm{H}) .{ }^{13} \mathrm{C}-\mathrm{NMR}(50.3 \mathrm{MHz}$, 
$\mathrm{CDCl}_{3}$, major isomer): $\delta=19.8,29.1,33.7,46.1,52.2,59.6,67.5,81.5,90.6,167.7,205.6$. IR (neat) $\tilde{v}=3496$ (br, w), 2954 (m), 2879 (w), 2109 (s), 1749 (s), 1719 (s), 1657 (w), 1629 (w), 1439 (m), 1401 (w), 1321 (m), 1260 (m), $1104(\mathrm{~m}), 1018(\mathrm{w}), 988(\mathrm{w}) \mathrm{cm}^{-1}$. MS (DCI, $\left.\mathrm{NH}_{3}\right): m / z(\%)=289\left(\left[\mathrm{M}+\mathrm{NH}_{4}\right]^{+}, 100\right), 271\left(\mathrm{M}^{+}, 2\right), 214(48), 164$ (20). Anal. calcd for $\mathrm{C}_{11} \mathrm{H}_{17} \mathrm{~N}_{3} \mathrm{O}_{5}$ (271.27): C 48.79, H 6.35, N 15.54; found: C 49.07, H 6.61, N 15.46.

Ethyl 4-(2-azido-1-hydroxycyclopentyl)-2-methyl-3-oxobutyrate (3i): The starting materials diisopropylamine $(1.05 \mathrm{~g}, 10.50 \mathrm{mmol}), n \mathrm{BuLi}(6.9 \mathrm{ml}, 10.05 \mathrm{mmol}, 15 \%$ solution in hexanes), 2-methyl-3-oxobutyrate (1i) (0.63 g, $4.40 \mathrm{mmol})$ and 2-azidocyclopentanone (2a) $(0.50 \mathrm{~g}, 4.00 \mathrm{mmol})$ yielded $3 \mathbf{i}$ as a yellow oil $(0.43 \mathrm{~g}, 40 \%, 3$ stereocenters, mixture of 2 diastereomers). ${ }^{1} \mathrm{H}-\mathrm{NMR}\left(250 \mathrm{MHz}, \mathrm{CDCl}_{3}\right.$, major isomer): $\delta=1.22\left(\mathrm{t},{ }^{3} \mathrm{~J}=7 \mathrm{~Hz}, 3 \mathrm{H}\right)$, $1.28\left(\mathrm{~d},{ }^{3} J=7 \mathrm{~Hz}, 3 \mathrm{H}\right) 1.42-2.02(\mathrm{~m}, 6 \mathrm{H}), 2.63\left(\mathrm{~d},{ }^{2} J=15 \mathrm{~Hz}, 1 \mathrm{H}\right), 2.90\left(\mathrm{~d},{ }^{2} J=15 \mathrm{~Hz}, 1\right.$ H), $3.30(\mathrm{~m}, 1 \mathrm{H}), 3.56\left(\mathrm{t},{ }^{3} \mathrm{~J}=7 \mathrm{~Hz}, 1 \mathrm{H}\right), 4.15\left(\mathrm{q},{ }^{3} J=7 \mathrm{~Hz}, 2 \mathrm{H}\right) .{ }^{13} \mathrm{C}-\mathrm{NMR}(50.3 \mathrm{MHz}$, $\mathrm{CDCl}_{3}$, major isomer): $\delta=12.3,13.9,19.1,26.7,35.8,48.2,53.6,61.4,67.5,79.9,170.0$, 206.5. IR (neat) $\tilde{v}=3510$ (br, w), 2980 (w), 2105 (s), 1741 (s), $1710(\mathrm{~s}), 1451(\mathrm{w}), 1398$ (w), 1378 (w), 1303 (w), 1257 (m), 1497 (m), 1075 (w), $1023(\mathrm{w}) \mathrm{cm}^{-1} . \mathrm{MS}\left(\mathrm{DCI}, \mathrm{NH}_{3}\right): \mathrm{m} / z(\%)$ $=556\left(\left[2 \mathrm{M}+\mathrm{NH}_{4}\right]^{+}, 80\right), 511(41), 287\left(\left[\mathrm{M}+\mathrm{NH}_{4}\right]^{+}, 100\right), 242(49), 224$ (11). Anal. calcd for $\mathrm{C}_{12} \mathrm{H}_{19} \mathrm{~N}_{3} \mathrm{O}_{4}$ (269.30): C 53.50, H 7.11, N 15.60; found: C 53.88, H 7.29, N 15.57.

Ethyl 4-(2-azido-1-hydroxycyclopentyl)-2-benzyl-3-oxobutyrate $\quad \mathbf{( 3 j}):$ The starting materials diisopropylamine $(0.52 \mathrm{~g}, 5.20 \mathrm{mmol}), n \mathrm{BuLi}(2.2 \mathrm{ml}, 5.20 \mathrm{mmol}, 23 \%$ solution in hexanes), ethyl 2-benzyl-3-oxo-butyrate (1j) (0.48 g, $2.20 \mathrm{mmol})$ and 2-azido-cyclopentanone (2a) $(0.25 \mathrm{~g}, 2.00 \mathrm{mmol})$ yielded $\mathbf{3 j}$ as a yellow oil $(0.22 \mathrm{~g}, 32 \%, 3$ stereocenters, mixture of 2 diastereomers). ${ }^{1} \mathrm{H}$ NMR (250 MHz, $\mathrm{CDCl}_{3}$, major isomer): $\delta=1.20\left(\mathrm{t},{ }^{3} \mathrm{~J}=7 \mathrm{~Hz}, 3 \mathrm{H}\right.$, $\left.\mathrm{CH}_{2} \mathrm{CH}_{3}\right), 1.42-2.01(\mathrm{~m}, 6 \mathrm{H}), 2.64\left(\mathrm{~d},{ }^{2} J=17 \mathrm{~Hz}, 1 \mathrm{H}\right), 2.97\left(\mathrm{~d},{ }^{2} J=17 \mathrm{~Hz}, 1 \mathrm{H}\right), 3.17(\mathrm{~m}$, 
$2 \mathrm{H}), 3.20(\mathrm{~m}, 1 \mathrm{H}), 3.84(\mathrm{~m}, 1 \mathrm{H}), 4.17\left(\mathrm{q},{ }^{3} J=7 \mathrm{~Hz}, 2 \mathrm{H}\right), 7.22(\mathrm{~m}, 5 \mathrm{H}) .{ }^{13} \mathrm{C}$ NMR $(50.3$ $\mathrm{MHz}, \mathrm{CDCl}_{3}$, major isomer): $\delta=14.0,19.2,27.0,33.9,35.9,49.5,61.5,61.7,67.5,79.9$, 126.9, 128.6, 128.8, 137.7, 170.1, 206.6. IR (neat): $\tilde{v}=3505,3517$ (br, w), 2976 (s), 2934 (m), 2871 (m), 2105 (s), 1741 (s), 1711 (s), 1495 (w), 1449 (m), 1373 (m), 1347 (m), 1300 (m), 1259 (s), 1209 (m), 1156 (m), 1116 (m), 1074 (m), 1029 (m), 749 (w), 701 (w) cm cm $^{-1}$ MS (DCI, NH $\left.\mathrm{NH}_{3}\right): m / z(\%)=363\left(\left(\mathrm{M}+\mathrm{NH}_{4}\right)^{+}, 100\right), 346\left((\mathrm{M}+\mathrm{H})^{+}, 14\right), 254(6), 226(2), 159$ (3).

Ethyl 4-(2-azido-1-hydroxycyclopentyl)-2-ethyl-3-oxobutyrate (3k): The starting materials diisopropylamine $(0.52 \mathrm{~g}, 5.20 \mathrm{mmol}), n \mathrm{BuLi}(2.2 \mathrm{ml}, 5.20 \mathrm{mmol}, 23 \%$ solution in hexanes), ethyl 2-ethyl-3-oxobutyrate (1k) (0.35 g, $2.20 \mathrm{mmol})$ and 2-azidocyclopentanone (2a) $(0.25 \mathrm{~g}, 2.00 \mathrm{mmol})$ yielded $\mathbf{3 k}$ as a yellow oil $(0.23 \mathrm{~g}, 41 \%, 3$ stereocenters, mixture of 2 diastereomers). ${ }^{1} \mathrm{H} \mathrm{NMR}\left(250 \mathrm{MHz}, \mathrm{CDCl}_{3}\right.$, major isomer): $\delta=0.93\left(\mathrm{t},{ }^{3} \mathrm{~J}=7 \mathrm{~Hz}, 3 \mathrm{H}\right)$, $1.27\left(\mathrm{t},{ }^{3} \mathrm{~J}=7 \mathrm{~Hz}, 3 \mathrm{H}\right), 1.57-2.05(\mathrm{~m}, 8 \mathrm{H}), 2.63\left(\mathrm{dd},{ }^{2} \mathrm{~J}=18 \mathrm{~Hz},{ }^{3} \mathrm{~J}=5 \mathrm{~Hz}, 1 \mathrm{H}\right), 2.97$ (dd, $\left.{ }^{2} J=17 \mathrm{~Hz},{ }^{3} J=5 \mathrm{~Hz}, 1 \mathrm{H}\right), 3.35\left(\mathrm{t},{ }^{3} J=7 \mathrm{~Hz}, 1 \mathrm{H}\right), 3.37(\mathrm{~m}, 1 \mathrm{H}), 4.18\left(\mathrm{q},{ }^{3} J=7 \mathrm{~Hz}, 2 \mathrm{H}\right)$. ${ }^{13} \mathrm{C} \mathrm{NMR}\left(75.5 \mathrm{MHz}, \mathrm{CDCl}_{3}\right.$, major isomer): $\delta=11.8,14.1,19.2,21.5,26.8,36.0,48.5$, 61.5, 61.5, $67.6\left(\mathrm{~N}_{3} \mathrm{CH}\right), 79.9,169.4,206.3 . \mathrm{IR}$ (neat): $\tilde{\mathrm{v}}=3504$ (br, w), 2973 (m), 2940 (m), 2879 (m), 2105 (s), 1739 (s), 1709 (s), 1461 (w), 1388 (w), 1372 (w), 1344 (w), 1297 (w), $1258(\mathrm{~m}), 1186(\mathrm{~m}), 1136(\mathrm{w}), 1097(\mathrm{w}), 1022(\mathrm{w}) \mathrm{cm}^{-1}$. MS (DCI, $\left.\mathrm{NH}_{3}\right): \mathrm{m} / \mathrm{z}(\%)=584$ $\left(\left(2 \mathrm{M}+\mathrm{NH}_{4}\right)^{+}, 1\right), 302\left(\left(\mathrm{M}+\mathrm{NH}_{4}+\mathrm{H}\right)^{+}, 13\right), 301\left(\left(\mathrm{M}+\mathrm{NH}_{4}\right)^{+}, 100\right), 284\left((\mathrm{M}+\mathrm{H})^{+}, 31\right), 256(17)$, 238 (18), $192(11), 122(2)$.

Ethyl 4-(2-azido-1-hydroxycyclopentyl)-2-n-butyl-3-oxobutyrate (3l): The starting materials diisopropylamine $(0.52 \mathrm{~g}, 5.20 \mathrm{mmol}), n \mathrm{BuLi}(2.2 \mathrm{ml}, 5.20 \mathrm{mmol}, 23 \%$ solution in hexane), ethyl 2-n-butyl-3-oxobutyrate (11) $(0.41 \mathrm{~g}, 2.20 \mathrm{mmol})$ and 2-azidocyclopentanone 
(2a) $(0.25 \mathrm{~g}, 2.00 \mathrm{mmol})$ yielded $3 \mathrm{l}$ as a yellow oil $(0.28 \mathrm{~g}, 45 \%, 3$ stereocenters, mixture of 2 diastereomers). ${ }^{1} \mathrm{H}$ NMR (250 MHz, $\mathrm{CDCl}_{3}$, major isomer): $\delta=0.89\left(\mathrm{t},{ }^{3} \mathrm{~J}=7 \mathrm{~Hz}, 3 \mathrm{H}\right), 1.21$ $-2.12(\mathrm{~m}, 12 \mathrm{H}), 1.27\left(\mathrm{t},{ }^{3} J=7 \mathrm{~Hz}, 3 \mathrm{H}\right), 2.60\left(\mathrm{dd},{ }^{2} J=18 \mathrm{~Hz},{ }^{3} J=5 \mathrm{~Hz}, 1 \mathrm{H}\right), 2.96\left(\mathrm{dd},{ }^{2} J\right.$ $\left.=17 \mathrm{~Hz},{ }^{3} J=13 \mathrm{~Hz}, 1 \mathrm{H}\right), 3.27\left(\mathrm{t},{ }^{3} J=7 \mathrm{~Hz}, 1 \mathrm{H}\right), 3.48\left(\mathrm{t},{ }^{3} J=7 \mathrm{~Hz}, 1 \mathrm{H}\right), 4.18\left(\mathrm{q},{ }^{3} J=7\right.$ $\mathrm{Hz}, 2 \mathrm{H}) .{ }^{13} \mathrm{C} \mathrm{NMR}\left(75.5 \mathrm{MHz}, \mathrm{CDCl}_{3}\right.$, major isomer): $\delta=13.8,14.1,19.2,19.2,22.4,26.8$, 27.7, 36.0, 48.5, 60.0, 61.5, 67.6, 78.0, 169.4, 206.4. IR (neat): $\tilde{v}=3512$ (br, w), 2960 (s), 2933 (s), 2873 (m), 2105 (s), 1739 (s), 1710 (s), 1465 (m), 1448 (m), 1371 (m), 1345 (m), $1298(\mathrm{~m}), 1255$ (m), $1219(\mathrm{~m}), 1181(\mathrm{~m}), 1117(\mathrm{~m}), 1075(\mathrm{w}), 1023(\mathrm{~m}) \mathrm{cm}^{-1}$. MS (DCI, $\left.\mathrm{NH}_{3}\right): m / \mathrm{z}(\%)=640\left(\left(2 \mathrm{M}+\mathrm{NH}_{4}\right)^{+}, 1\right), 330\left(\left(\mathrm{M}+\mathrm{NH}_{4}+\mathrm{H}\right)^{+}, 32\right), 329\left(\left(\mathrm{M}+\mathrm{NH}_{4}\right)^{+}, 100\right), 312$ $\left((\mathrm{M}+\mathrm{H})^{+}, 23\right), 284(13), 266(8)$.

5-(2-Azido-1-hydroxycyclopentyl)-4-hydroxypent-3-en-2-one (3m): The starting materials diisopropylamine (0.52 g, $5.20 \mathrm{mmol}), n \mathrm{BuLi}(2.2 \mathrm{ml}, 5.20 \mathrm{mmol}, 23 \%$ solution in hexanes), acetylacetone (1m) (0.22 g, $2.20 \mathrm{mmol})$ and 2-azidocyclopentanone (2a) (0.25 g, $2.00 \mathrm{mmol})$ yielded $\mathbf{3 m}$ as a yellow oil $(0.21 \mathrm{~g}, 48 \%$, enol-form, $\mathrm{dr}=10: 1) .{ }^{1} \mathrm{H} \mathrm{NMR}\left(300 \mathrm{MHz}, \mathrm{CDCl}_{3}\right.$, major isomer): $\delta=1.51-2.07(\mathrm{~m}, 6 \mathrm{H}), 1.97(\mathrm{~s}, 3 \mathrm{H}), 2.33\left(\mathrm{~d},{ }^{2} J=16 \mathrm{~Hz}, 1 \mathrm{H}\right), 2.52\left(\mathrm{~d},{ }^{2} J=\right.$

$16 \mathrm{~Hz}, 1 \mathrm{H}), 3.29\left(\mathrm{t},{ }^{3} \mathrm{~J}=6 \mathrm{~Hz}, 1 \mathrm{H}\right), 5.40(\mathrm{~s}, 1 \mathrm{H}) .{ }^{13} \mathrm{C} \mathrm{NMR}\left(50.3 \mathrm{MHz}, \mathrm{CDCl}_{3}\right.$, major isomer): $\delta=19.3,21.2,27.6,36.1,47.0,68.2,80.6,101.3,190.9,192.2$. IR (neat): $\tilde{v}=3442$ (br, w), 2975 (m), 2937 (m), 2872 (m), 2105 (s), 1723 (w), 1708 (w), 1617 (m), 1444 (m), 1419 (m), 1382 (m), 1354 (m), 1300 (m), 1258 (m), 1179 (w), 1149 (m), $1117(\mathrm{~m}), 1076(\mathrm{w})$, $1025(\mathrm{w}) \mathrm{cm}^{-1}$. MS (DCI, $\left.\mathrm{NH}_{3}\right): m / z(\%)=468\left(\left(2 \mathrm{M}+\mathrm{NH}_{4}\right)^{+}, 1\right), 243\left(\left(\mathrm{M}+\mathrm{NH}_{4}\right)^{+}, 100\right), 226$ $\left((\mathrm{M}+\mathrm{H})^{+}, 93\right), 182(76), 178(41), 159(17), 142(5)$.

6-(2Azido-1-hydroxycyclopentyl)-5-hydroxy-2,2-dimethylhex-4-en-3-one $\quad$ (3n): The starting materials diisopropylamine $(0.52 \mathrm{~g}, 5.20 \mathrm{mmol}), n \mathrm{BuLi}(2.2 \mathrm{ml}, 5.20 \mathrm{mmol}, 23 \%$ 
solution in hexanes), 5,5-dimethylhexane-2,4-dione (1n) $(0.22 \mathrm{~g}, 2.20 \mathrm{mmol})$ and 2 azidocyclopentanone (2a) $(0.25 \mathrm{~g}, 2.00 \mathrm{mmol})$ yielded $\mathbf{3 n}$ as a yellow oil $(0.20 \mathrm{~g}, 38 \%$, enolform, $\mathrm{dr}=2: 1) .{ }^{1} \mathrm{H} \mathrm{NMR}\left(250 \mathrm{MHz}, \mathrm{CDCl}_{3}\right.$, major isomer): $\delta=1.18(\mathrm{~s}, 9 \mathrm{H}), 1.53-2.08(\mathrm{~m}$, $\left.6 \mathrm{H},\left(\mathrm{CH}_{2}\right)_{3}\right), 2.47\left(\mathrm{~d},{ }^{2} J=16 \mathrm{~Hz}, 1 \mathrm{H}, \mathrm{CH} \mathrm{H}_{2} \mathrm{COH}\right), 2.67\left(\mathrm{~d},{ }^{2} J=16 \mathrm{~Hz}, 1 \mathrm{H}\right), 3.42\left(\mathrm{t},{ }^{3} \mathrm{~J}=6\right.$ $\mathrm{Hz}, 1 \mathrm{H}), 5.64(\mathrm{~s}, 1 \mathrm{H}) .{ }^{13} \mathrm{C} \mathrm{NMR}\left(50.3 \mathrm{MHz}, \mathrm{CDCl}_{3}\right.$, major isomer): $\delta=19.3,27.2,27.5$, 36.1, 44.0, 46.8, 67.9, 80.5, 82.4, 96.9, 194.4. IR (neat): $\tilde{v}=3473$ (br, w), 2969 (m), 2106 (s), 1607 (s), 1462 (m), 1437 (m), 1297 (m), $1264(\mathrm{~m}), 1221$ (w), 1138 (w), 1098 (w), 1062 (w), 1023 (w), 944 (w), 924 (w), $883(w), 791(w) \mathrm{cm}^{-1}$. MS (DCI, $\left.\mathrm{NH}_{3}\right): \mathrm{m} / \mathrm{z}(\%)=552$ $\left(\left(2 \mathrm{M}+\mathrm{NH}_{4}\right)^{+}, 65\right), 285\left(\left(\mathrm{M}+\mathrm{NH}_{4}\right)^{+}, 100\right), 268\left((\mathrm{M}+\mathrm{H})^{+}, 88\right), 222(17)$.

6-(2-Azido-1-hydroxycyclopentyl)-5-hydroxyhept-4-en-3-one (3o): The starting materials diisopropylamine (0.52 g, $5.20 \mathrm{mmol}), n \mathrm{BuLi}(2.2 \mathrm{ml}, 5.20 \mathrm{mmol}, 23 \%$ solution in hexanes), heptane-3,5-dione (1o) (0.28 g, $2.20 \mathrm{mmol})$ and 2-azidocyclopentanone (2a) (0.25 g, 2.00 mmol) yielded 3o as a yellow oil (0.13 g, 25\%, enol-form, 3 stereocenters, 2 diastereomers). ${ }^{1} \mathrm{H} \mathrm{NMR} \mathrm{(250} \mathrm{MHz,} \mathrm{CDCl}_{3}$, major isomer): $\delta=1.14\left(\mathrm{t},{ }^{3} J=7 \mathrm{~Hz}, 3 \mathrm{H}\right), 1.23\left(\mathrm{~d},{ }^{3} J=7 \mathrm{~Hz}, 3\right.$ H), $1.50-2.07(\mathrm{~m}, 6 \mathrm{H}), 2.35\left(\mathrm{q},{ }^{3} J=7 \mathrm{~Hz}, 2 \mathrm{H}\right), 3.47\left(\mathrm{q},{ }^{3} J=7 \mathrm{~Hz}, 1 \mathrm{H}\right), 3.69\left(\mathrm{t},{ }^{3} J=7 \mathrm{~Hz}\right.$, $1 \mathrm{H}), 5.52(\mathrm{~s}, 1 \mathrm{H}) .{ }^{13} \mathrm{C} \mathrm{NMR}\left(50.3 \mathrm{MHz}, \mathrm{CDCl}_{3}\right.$, major isomer): $\delta=9.6,13.6,19.4,28.6$, 31.4, 35.0, 49.2, 67.6, 82.9, 98.9, 195.9, 197.4. IR (neat): $\tilde{v}=3422$ (br, m), 3417 (br, m), 2977 (s), 2932 (s), 2877 (s), 2106 (s), 1721 (m), 1613 (s), 1457 (m), 1412 (w), 1379 (m), 1345 (m), 1324 (m), 1298 (m), 1259 (m), 1179 (m), 1121 (s), 1104 (s), 1048 (m), 1011 (m) $\mathrm{cm}^{-1}$. MS (DCI, $\left.\mathrm{NH}_{3}\right): \mathrm{m} / z(\%)=524\left(\left(2 \mathrm{M}+\mathrm{NH}_{4}\right)^{+}, 6\right), 271\left(\left(\mathrm{M}+\mathrm{NH}_{4}\right)^{+}, 59\right), 254\left((\mathrm{M}+\mathrm{H})^{+}\right.$, 100), 226 (30), 208 (8).

4-(2-Azido-1-hydroxycyclopentyl)-3-hydroxy-1-phenylbut-2-en-1-one (3p): The starting materials diisopropylamine $(0.52 \mathrm{~g}, 5.20 \mathrm{mmol}), n \mathrm{BuLi}(2.2 \mathrm{ml}, 5.20 \mathrm{mmol}, 23 \%$ solution in 
hexane), benzoylacetone (1p) $(0.36 \mathrm{~g}, 2.20 \mathrm{mmol})$ and 2-azidocyclopentanone (2a) (0.25 g, $2.00 \mathrm{mmol})$ yielded $3 \mathbf{p}$ as a yellow oil $(0.09 \mathrm{~g}, 15 \%$, enol-form, $\mathrm{dr}=10: 1) .{ }^{1} \mathrm{H}$ NMR (250 $\mathrm{MHz}, \mathrm{CDCl}_{3}$, major isomer): $\delta=1.61-2.15(\mathrm{~m}, 6 \mathrm{H}), 2.59\left(\mathrm{~d},{ }^{2} \mathrm{~J}=12 \mathrm{~Hz}, 1 \mathrm{H}\right), 2.81\left(\mathrm{~d},{ }^{2} J=\right.$ $12 \mathrm{~Hz}, 1 \mathrm{H}), 3.47\left(\mathrm{t},{ }^{3} J=7 \mathrm{~Hz}, 1 \mathrm{H}\right), 6.22(\mathrm{~s}, 1 \mathrm{H}), 7.48(\mathrm{~m}, 3 \mathrm{H}), 7.88\left(\mathrm{~d},{ }^{3} J=5 \mathrm{~Hz}, 2 \mathrm{H}\right)$. ${ }^{13} \mathrm{C}$ NMR (50.3 MHz, $\mathrm{CDCl}_{3}$, major isomer): $\delta=19.4,27.5,36.1,47.2,67.9,80.6,97.8$, 127.1, 128.7, 132.6, 134.2, 182.8, 195.2. MS (DCI, $\left.\mathrm{NH}_{3}\right): m / z(\%)=592\left(\left(2 \mathrm{M}+\mathrm{NH}_{4}\right)^{+}, 19\right)$, $322\left(\left(\mathrm{M}+\mathrm{NH}_{3}+\mathrm{NH}_{4}\right)^{+}, 15\right), 305\left(\left(\mathrm{M}+\mathrm{NH}_{4}\right)^{+}, 100\right), 288\left((\mathrm{M}+\mathrm{H})^{+}, 85\right), 245(27)$.

3-(2-Azido-1-hydroxycyclopentyl)-1-tosylpropan-2-one (3q). To a THF solution (100 ml) of diisopropylamine $(3.68 \mathrm{~g}, 36.4 \mathrm{mmol})$ was added a $1.6 \mathrm{M}$ solution of $n \mathrm{BuLi}$ in hexane $(22.8 \mathrm{ml}, 36.5 \mathrm{mmol})$ at $0{ }^{\circ} \mathrm{C}$. After stirring for 20 minutes, 1-tosylpropan-2-one (2.89 $\mathrm{g}, 13.6$ mmol) was added and the solution was stirred for $1 \mathrm{~h}$ at $0{ }^{\circ} \mathrm{C}$. 2-Azidocyclopentanone (1.70 $\mathrm{g}, 13.6 \mathrm{mmol})$ was added at $-78{ }^{\circ} \mathrm{C}$ and the reaction mixture was warmed to ambient temperature within $21 \mathrm{~h}$. A saturated solution of $\mathrm{NH}_{4} \mathrm{Cl}(100 \mathrm{ml})$ was added and the organic layer was separated. The organic layer was extracted with $\mathrm{Et}_{2} \mathrm{O}(2 \times 50 \mathrm{ml})$ and $\mathrm{CH}_{2} \mathrm{Cl}_{2}(3 \mathrm{x}$ $50 \mathrm{ml})$. The combined organic layers were washed with brine, dried $\left(\mathrm{Na}_{2} \mathrm{SO}_{4}\right)$, filtered, and the solvent of the filtrate was removed in vacuo. The residue was purified by chromatography ( ilica gel, hexane/ethylacetate $=3 / 1)$ to give $\mathbf{3 q}$ as a brownish oil $(1.06 \mathrm{~g}, 23 \%$, $\mathrm{dr}=10: 1)$. An analytical sample of the pure cis-diastereomer could be separated. ${ }^{1} \mathrm{H}$ NMR $\left(\mathrm{CDCl}_{3}, 300\right.$ MHz): $\delta=1.50-1.75(\mathrm{~m}, 2 \mathrm{H}), 1.75-2.10(\mathrm{~m}, 4 \mathrm{H}), 2.46(\mathrm{~s}, 3 \mathrm{H}), 2.83\left(\mathrm{~d},{ }^{2} J=16.4 \mathrm{~Hz}, 1 \mathrm{H}\right)$, $3.14\left(\mathrm{~d},{ }^{2} J=16.4 \mathrm{~Hz}, 1 \mathrm{H}\right), 3.37\left(\mathrm{dd},{ }^{3} J_{1}={ }^{3} J_{2}=8.4 \mathrm{~Hz}, 1 \mathrm{H}\right), 4.17\left(\mathrm{~d},{ }^{2} J=13.1 \mathrm{~Hz}, 1 \mathrm{H}\right)$, $4.31\left(\mathrm{~d},{ }^{2} J=13.1 \mathrm{~Hz}, 1 \mathrm{H}\right), 7.38\left(\mathrm{~d},{ }^{3} J=8.2 \mathrm{~Hz}, 2 \mathrm{H}\right), 7.76\left(\mathrm{~d},{ }^{3} J=8.2 \mathrm{~Hz}, 2 \mathrm{H}\right) .{ }^{13} \mathrm{C}$ NMR $\left(\mathrm{CDCl}_{3}, 75 \mathrm{MHz}\right): \delta=19.2,21.6,27.0,35.9,51.4,67.7,68.0,79.8,128.2,130.0,135.5$, 145.6, 198.5 . 
Ethyl 4-(2-azido-1-hydroxyindan-1-yl)-3-oxobutyrate (3r): The starting materials diisopropylamine (0.52 g, $5.20 \mathrm{mmol}), n \mathrm{BuLi}(2.2 \mathrm{ml}, 5.20 \mathrm{mmol}, 23 \%$ solution in hexane), ethyl acetoacetate (1a) $(0.29 \mathrm{~g}, 2.20 \mathrm{mmol})$ and 1-azidoindan-2-one (2b) (0.55 g, 2.00 mmol) yielded $3 \mathbf{r}$ as a yellow oil $(0.34 \mathrm{~g}, 63 \%, \mathrm{dr}=10: 1) .{ }^{1} \mathrm{H}$ NMR $\left(250 \mathrm{MHz}, \mathrm{CDCl}_{3}\right.$, major isomer): $\delta=1.28\left(\mathrm{t},{ }^{3} J=7 \mathrm{~Hz}, 3 \mathrm{H}\right), 2.92\left(\mathrm{~d},{ }^{2} J=16 \mathrm{~Hz}, 1 \mathrm{H}\right), 3.09\left(\mathrm{~d},{ }^{2} J=16 \mathrm{~Hz}, 1\right.$ H), $3.16(\mathrm{~m}, 2 \mathrm{H}), 3.59(\mathrm{~s}, 2 \mathrm{H}), 4.17(\mathrm{~m}, 1 \mathrm{H}), 4.20\left(\mathrm{q},{ }^{3} \mathrm{~J}=7 \mathrm{~Hz}, 2 \mathrm{H}\right), 7.20-7.37(\mathrm{~m}, 4 \mathrm{H})$. ${ }^{13} \mathrm{C}$ NMR (50.3 MHz, $\mathrm{CDCl}_{3}$, major isomer): $\delta=14.1,34.8,49.5,50.72,61.6,67.7,82.3$, 123.4, 125.3, 127.7, 129.3, 138.5, 143.7, 166.9, 202.8. IR (neat): $\tilde{v}=3474$ (br, m), 2983 (m), 2935 (m), 2108 (s), 1741 (s), 1714 (s), 1643 (m), 1569 (m), 1474 (m), 1463 (m), 1440 (m), 1406 (s), 1368 (s), 1320 (s), 1264 (s), 1177 (s), 1158 (s), 1095 (m), 1028 (s), 976 (w), $766(\mathrm{~m}), 558(\mathrm{w}) \mathrm{cm}^{-1}$. MS (DCI, $\left.\mathrm{NH}_{3}\right): \mathrm{m} / z(\%)=624\left(\left(2 \mathrm{M}+\mathrm{NH}_{4}\right)^{+}, 24\right), 321\left(\left(\mathrm{M}+\mathrm{NH}_{4}\right)^{+}\right.$, 100), 258 (84), 212 (14).

5-(2-Azido-1-hydroxyindan-1-yl)-4-hydroxypent-3-en-2-one (3s): The starting materials diisopropylamine (0.52 g, $5.20 \mathrm{mmol}), n \mathrm{BuLi}(2.2 \mathrm{ml}, 5.20 \mathrm{mmol}, 23 \%$ solution in hexane), acetylacetone (1m) (0.29 g, $2.20 \mathrm{mmol})$ and 1-azidoindan-2-one (2b) (0.55 g, $2.00 \mathrm{mmol})$ yielded $3 \mathrm{~s}$ as a yellow oil $(0.26 \mathrm{~g}, 54 \%$, enol-form, $\mathrm{dr}=2: 1) .{ }^{1} \mathrm{H} \mathrm{NMR}\left(250 \mathrm{MHz}, \mathrm{CDCl}_{3}\right.$, major isomer): $\delta=2.07(\mathrm{~s}, 3 \mathrm{H}), 2.66\left(\mathrm{~d},{ }^{2} J=16 \mathrm{~Hz}, 1 \mathrm{H}\right), 2.87\left(\mathrm{~d},{ }^{2} J=16 \mathrm{~Hz}, 1 \mathrm{H}\right), 3.16(\mathrm{~m}$, $2 \mathrm{H}), 4.17\left(\mathrm{t},{ }^{3} \mathrm{~J}=5 \mathrm{~Hz}, 1 \mathrm{H}\right), 5.58(\mathrm{~s}, 1 \mathrm{H}), 7.21-7.39(\mathrm{~m}, 4 \mathrm{H}) .{ }^{13} \mathrm{C}$ NMR $(50.3 \mathrm{MHz}$, $\mathrm{CDCl}_{3}$, major isomer): $\delta=24.3,35.0,46.2,67.8,82.6,102.0,123.5,125.1,127.6,129.2$, 138.6, 143.8, 190.3, 192.7. IR (neat): $\widetilde{v}=3415$ (br, w), 2973 (w), 2926 (w), 2856 (w), 2107 (s), 1723 (m), 1700 (m), 1661 (s), 1613 (s), 1477 (m), 1460 (m), 1433 (m), 1390 (s), 1356 (s), 1318 (m), 1302 (m), 1268 (s), 1176 (m), 1155 (m), $1114(\mathrm{~m}), 1076$ (w), 1023 (m), 994 (m), $950(w), 803(w), 764(m), 721(w), 553(w) \mathrm{cm}^{-1}$. MS (DCI, $\left.\mathrm{NH}_{3}\right): m / \mathrm{z}(\%)=564$ 
$\left(\left(2 \mathrm{M}+\mathrm{NH}_{4}\right)^{+}, 3\right), 511(16), 291\left(\left(\mathrm{M}+\mathrm{NH}_{4}\right)^{+}, 53\right), 273\left(\left(\mathrm{M}-\mathrm{H}_{2} \mathrm{O}+\mathrm{NH}_{4}\right)^{+}, 100\right), 256(53), 228$ (29), $144(63)$.

6-(2-Azido-1-hydroxyindan-1-yl)-5-hydroxy-2,2-dimethylhex-4-en-3-one $\quad(3 t): \quad$ The starting materials diisopropylamine $(0.30 \mathrm{~g}, 3.04 \mathrm{mmol}), n \mathrm{BuLi}(1.3 \mathrm{ml}, 3.04 \mathrm{mmol}, 23 \%$ solution in hexane), 5,5-dimethylhexane-2,4-dione (1n) $(0.18 \mathrm{~g}, 1.28 \mathrm{mmol})$ and 1azidoindan-2-one (2b) $(0.20 \mathrm{~g}, 1.16 \mathrm{mmol})$ yielded $\mathbf{3 t}$ as a yellow oil $(0.22 \mathrm{~g}, 61 \%, \mathrm{dr}=5: 1)$. ${ }^{1} \mathrm{H}$ NMR (250 MHz, $\mathrm{CDCl}_{3}$, major isomer): $\delta=1.19(\mathrm{~s}, 9 \mathrm{H}), 2.73\left(\mathrm{~d},{ }^{2} \mathrm{~J}=16 \mathrm{~Hz}, 1 \mathrm{H}\right), 2.92$ $\left(\mathrm{d},{ }^{2} J=16 \mathrm{~Hz}, 1 \mathrm{H}\right), 3.15(\mathrm{~m}, 2 \mathrm{H}), 4.17\left(\mathrm{t},{ }^{3} J=5 \mathrm{~Hz}, 1 \mathrm{H}\right), 5.61(\mathrm{~s}, 1 \mathrm{H}), 7.22-7.40(\mathrm{~m}, 4$ H). ${ }^{13} \mathrm{C}$ NMR (75.5 MHz, $\mathrm{CDCl}_{3}$, major isomer): $\delta=27.2,35.1,38.8,46.7,68.1,82.7,97.3$, 123.5, 125.1, 127.6, 129.2, 138.6, 143.8, 193.7, 199.6. IR (neat): $\tilde{v}=3442$ (br, w), 2969 (m), 2932 (m), $2871(\mathrm{w}), 2107$ (s), 1712 (m), 1698 (m), 1602 (s), 1478 (m), 1461 (m), 1435 (m), 1396 (m), 1364 (m), 1320 (m), 1268 (s), 1221 (m), 1181 (m), 1143 (m), 1119 (m), 1078 (w), $1054(\mathrm{w}), 1022(\mathrm{w}) \mathrm{cm}^{-1}$. MS (DCI, $\left.\mathrm{NH}_{3}\right): \mathrm{m} / z(\%)=648\left(\left(2 \mathrm{M}+\mathrm{NH}_{4}\right)^{+}, 22\right), 459(27)$, $333\left(\left(\mathrm{M}+\mathrm{NH}_{4}\right)^{+}, 91\right), 270(100), 144(80)$.

4-(2-Azido-1-hydroxyindan-1-yl)-3-hydroxy-1-phenylbut-2-en-1-one (3u): The starting materials diisopropylamine $(0.30 \mathrm{~g}, 3.04 \mathrm{mmol}), n \mathrm{BuLi}(1.3 \mathrm{ml}, 3.04 \mathrm{mmol}, 23 \%$ solution in hexane), benzoylacetone (1p) $(0.21 \mathrm{~g}, 1.28 \mathrm{mmol})$ and 1-azidoindan-2-one (2b) (0.20 g, $1.16 \mathrm{mmol})$ yielded $3 \mathbf{u}$ as a brownish oil $(0.21 \mathrm{~g}, 55 \%$, enol-form, $\mathrm{dr}=5: 1) .{ }^{1} \mathrm{H}$ NMR $(250$ $\mathrm{MHz}, \mathrm{CDCl}_{3}$, major isomer): $\delta=2.82\left(\mathrm{~d},{ }^{2} J=16 \mathrm{~Hz}, 1 \mathrm{H}\right), 3.03\left(\mathrm{~d},{ }^{2} J=16 \mathrm{~Hz}, 1 \mathrm{H}\right), 3.18$ $(\mathrm{m}, 2 \mathrm{H}), 4.24\left(\mathrm{t},{ }^{3} \mathrm{~J}=5 \mathrm{~Hz}, 1 \mathrm{H}\right), 6.25(\mathrm{~s}, 1 \mathrm{H}), 7.19-8.01(\mathrm{~m}, 9 \mathrm{H}) .{ }^{13} \mathrm{C}$ NMR $(50.3 \mathrm{MHz}$, $\mathrm{CDCl}_{3}$, major isomer): $\delta=35.0,47.1,68.0,82.7,98.2,123.5,125.2,127.1,127.6,128.7$, 129.2, 132.7, 134.0, 138.6, 143.7, 182.5, 194.9. IR (neat): $\tilde{v}=3430$ (br, m), 2972 (w), 2927 (w), 2106 (s), 1708 (m), 1672 (m), 1606 (s), 1570 (s), 1487 (m), 1460 (s), 1423 (m), 1320 
(m), 1268 (s), 1183 (m), 1152 (m), 1113 (m), 1101 (m), 1075 (m), 1025 (w), 1000 (w), 975 (m), $947(\mathrm{w}), 765(\mathrm{~s}), 724(\mathrm{w}), 697(\mathrm{~m}), 569(\mathrm{w}) \mathrm{cm}^{-1}$. MS (DCI, $\left.\mathrm{NH}_{3}\right): \mathrm{m} / z(\%)=688$ $\left(\left(2 \mathrm{M}+\mathrm{NH}_{4}\right)^{+}, 12\right), 479(16), 353\left(\left(\mathrm{M}+\mathrm{NH}_{4}\right)^{+}, 93\right), 336\left((\mathrm{M}+\mathrm{H})^{+}, 100\right), 293(68), 180(24)$, $144(81)$.

7-Azido-4,6-dihydroxy-6-methylhept-3-en-2-one (3v): The starting materials diisopropylamine (0.92 g, $9.20 \mathrm{mmol}), n \mathrm{BuLi}(3.8 \mathrm{ml}, 9.20 \mathrm{mmol}, 23 \%$ solution in hexane), acetyl acetone (1m) (0.39 g, $3.90 \mathrm{mmol})$ and 1-azidopropan-2-one (2c) (0.35 g, $3.50 \mathrm{mmol})$ yielded $3 \mathrm{v}$ as a yellow oil $(0.39 \mathrm{~g}, 60 \%) .{ }^{1} \mathrm{H}$ NMR $\left(250 \mathrm{MHz}, \mathrm{CDCl}_{3}\right): \delta=1.25(\mathrm{~s}, 3 \mathrm{H}), 2.05$ (s, $3 \mathrm{H}), 2.46\left(\mathrm{~d},{ }^{2} J=17 \mathrm{~Hz}, 1 \mathrm{H}\right), 2.60\left(\mathrm{~d},{ }^{2} J=17 \mathrm{~Hz}, 1 \mathrm{H}\right), 3.24\left(\mathrm{~d},{ }^{2} J=15 \mathrm{~Hz}, 1 \mathrm{H}\right), 3.32$ $\left(\mathrm{d},{ }^{2} J=15 \mathrm{~Hz}, 1 \mathrm{H}\right), 3.78(\mathrm{br}, 1 \mathrm{H}), 5.52(\mathrm{~s}, 1 \mathrm{H}) .{ }^{13} \mathrm{C}$ NMR $\left(50.3 \mathrm{MHz}, \mathrm{CDCl}_{3}\right): \delta=24.0$, 25.3, 46.3, 60.0, 72.6, 101.8, 189.5, 194.7. IR (neat) $\tilde{v}=3438$ (br, m), $2927(\mathrm{w}), 2105$ (s), $1721(\mathrm{~m}), 1701$ (m), 1617 (s), 1438 (m), $1351(\mathrm{~m}), 1296(\mathrm{~m}), 1150(\mathrm{w}), 935(\mathrm{w}) \mathrm{cm}^{-1} . \mathrm{MS}$ $\left(\right.$ DCI, $\left.\mathrm{NH}_{3}\right): m / z(\%)=416\left(\left(2 \mathrm{M}+\mathrm{NH}_{4}\right)^{+}, 8\right), 234\left(\left(\mathrm{M}+\mathrm{NH}_{3}+\mathrm{NH}_{4}\right)^{+}, 32\right), 217\left(\left(\mathrm{M}+\mathrm{NH}_{4}\right)^{+}\right.$, 100), $200\left((\mathrm{M}+\mathrm{H})^{+}, 45\right), 177(17), 171(25), 154(44)$.

6-Azido-3,5-dihydroxy-5-methyl-1-phenylhept-2-en-1-one (3w): The starting materials diisopropylamine (0.68 g, $6.80 \mathrm{mmol}), n \mathrm{BuLi}(2.9 \mathrm{ml}, 6.80 \mathrm{mmol}, 23 \%$ solution in hexane), benzoylacetone (1p) (0.47 g, $2.90 \mathrm{mmol})$ and 3-azidobutan-2-one (2d) (0.29 g, $2.60 \mathrm{mmol})$ yielded $3 \mathbf{w}$ as a yellow oil (0.34 g, 52\%, diastereomeric mixture). ${ }^{1} \mathrm{H}$ NMR (250 MHz, $\mathrm{CDCl}_{3}$, major isomer): $\delta=1.23(\mathrm{~s}, 3 \mathrm{H}), 1.33\left(\mathrm{~d},{ }^{3} J=7 \mathrm{~Hz}, 3 \mathrm{H}\right), 2.59\left(\mathrm{~d},{ }^{2} J=15 \mathrm{~Hz}, 1 \mathrm{H}\right)$, $2.82\left(\mathrm{~d},{ }^{2} J=15 \mathrm{~Hz}, 1 \mathrm{H}\right), 3.57(\mathrm{~m}, 1 \mathrm{H}), 6.22(\mathrm{~s}, 1 \mathrm{H}), 7.52(\mathrm{~m}, 3 \mathrm{H}), 7.89\left(\mathrm{~d},{ }^{3} J=6 \mathrm{~Hz}, 2\right.$ H). ${ }^{13} \mathrm{C} \mathrm{NMR} \mathrm{(50.3} \mathrm{MHz,} \mathrm{CDCl}_{3}$, major isomer): $\delta=13.5,22.7,46.3,64.1,74.6,98.2$, 127.1, 128.7, 132.7, 133.79, 181.8, 197.6. IR (neat): $\tilde{v}=3477,3461$ (br, m), 2980 (m), 2938 (w), 2096 (s), 1707 (m), 1672 (m), 1605 (s), 1573 (s), 1491 (m), 1459 (m), 1423 (m), 
$1380(\mathrm{~m}), 1266$ (s), $1183(\mathrm{~m}), 1157$ (m), 1078 (m), $1004(\mathrm{w}), 944(\mathrm{w}), 766(\mathrm{~m}), 698(\mathrm{~m})$ $\mathrm{cm}^{-1}$. MS (DCI, NH $\left.\mathrm{NH}_{3}\right): m / z(\%)=568\left(\left(2 \mathrm{M}+\mathrm{NH}_{4}\right)^{+}, 1\right), 293\left(\left(\mathrm{M}+\mathrm{NH}_{4}\right)^{+}, 18\right), 276\left((\mathrm{M}+\mathrm{H})^{+}\right.$, 34), 248 (21), 232 (34), 222 (100), 205 (35), 147 (23).

Ethyl 4-(2-azido-1-hydroxycyclohexyl)-3-oxobutyrate $\mathbf{( 3 x )}:{ }^{6}$ The starting materials diisopropylamine (0.76 g, $7.6 \mathrm{mmol}), n \mathrm{BuLi}(5.0 \mathrm{ml}, 7.6 \mathrm{mmol}, 15 \%$ solution in hexane), ethyl acetoacetate (1a) $(0.41 \mathrm{~g}, 3.20 \mathrm{mmol})$ and 2-azidocyclohexanone (2e) $(0.40 \mathrm{~g}, 2.90$ mmol) yielded 3x as a yellow oil (0.75 g, 97\%, diastereomeric mixture). ${ }^{1} \mathrm{H}-\mathrm{NMR}(300 \mathrm{MHz}$, $\mathrm{CDCl}_{3}$, major isomer) $\delta=1.20-2.03(\mathrm{~m}, 8 \mathrm{H}), 1.26\left(\mathrm{t},{ }^{3} \mathrm{~J}=7 \mathrm{~Hz}, 3 \mathrm{H}\right) 2.58\left(\mathrm{~d},{ }^{2} J=18 \mathrm{~Hz}, 1\right.$ H), $3.04\left(\mathrm{~d},{ }^{2} J=18 \mathrm{~Hz}, 1 \mathrm{H}\right), 3.42\left(\mathrm{t},{ }^{3} J=6 \mathrm{~Hz}, 1 \mathrm{H}\right), 3.48(\mathrm{~s}, 2 \mathrm{H}), 4.18\left(\mathrm{q},{ }^{3} J=7 \mathrm{~Hz}, 2 \mathrm{H}\right)$. ${ }^{13} \mathrm{C}-\mathrm{NMR}\left(50.3 \mathrm{MHz}, \mathrm{CDCl}_{3}\right.$, major isomer): $\delta=14.0,20.3,24.0,25.6,36.0,50.3,50.9$, 61.5, 65.2, 73.5, 166.8, 204.1. IR (neat) $\tilde{v}=3497$ (br, w), 2940 (m), 2103 (s), 1740 (s), 1708 (m), 1447 (w), 1368 (w), 1316 (w), 1262 (m), 1159 (w), 1029 (w), 994 (w) cm ${ }^{-1}$. MS (DCI): $m / z(\%)=556\left(\left[2 \mathrm{M}+\mathrm{NH}_{4}\right]^{+}, 50\right), 549(12), 511(12), 288(14), 287\left(\left[\mathrm{M}+\mathrm{NH}_{4}\right]^{+}, 100\right), 270$ $\left([\mathrm{M}+\mathrm{H}]^{+}, 5\right), 242(12)$.

2-Azido-1-hydroxy-1-(2-oxo-1-propyl)cyclopentane (3y): To a $\mathrm{CH}_{2} \mathrm{Cl}_{2}$ solution (10 ml) of 2-azidocyclopentanone $\quad\left(\begin{array}{llll}0.20 \quad \mathrm{~g}, & 1.60 \quad \mathrm{mmol}) & \text { and } & \text { of }\end{array}\right.$ 2-(trimethylsilyloxy)propene $(0.23 \mathrm{~g}, 1.76 \mathrm{mmol})$ was slowly added $\mathrm{TiCl}_{4}(0.55 \mathrm{~g}, 2.88$ mmol) at $-78{ }^{\circ} \mathrm{C}$ and the solution was warmed to $20{ }^{\circ} \mathrm{C}$ within $18 \mathrm{~h}$. To the solution was added brine $(50 \mathrm{ml})$. The organic and the aqueous layer were separated and the latter was extracted with $\mathrm{CH}_{2} \mathrm{Cl}_{2}(3 \times 30 \mathrm{ml})$. The combined organic layers were dried $\left(\mathrm{Na}_{2} \mathrm{SO}_{4}\right)$, filtered and the solvent of the filtrate was removed in vacuo. The residue was purified by chromatography (silica gel, petroleum ether/ether $=4: 1-3: 1)$ to give $\mathbf{3 y}(0.17 \mathrm{~g}, 60 \%)$ as a yellow oil. ${ }^{1} \mathrm{H}$ NMR $\left(300 \mathrm{MHz}, \mathrm{CDCl}_{3}\right): \delta=1.57-2.08(\mathrm{~m}, 6 \mathrm{H}), 2.20(\mathrm{~s}, 3 \mathrm{H}), 2.53\left(\mathrm{~d},{ }^{2} J=\right.$ 
$17 \mathrm{~Hz}, \mathrm{~A}$ of $\mathrm{AB}, 1 \mathrm{H}), 2.88\left(\mathrm{~d},{ }^{2} J=17 \mathrm{~Hz}, \mathrm{~B}\right.$ of $\left.\mathrm{AB}, 1 \mathrm{H}\right), 3.24\left(\mathrm{t},{ }^{3} J=8 \mathrm{~Hz}, 1 \mathrm{H}\right),{ }^{13} \mathrm{C} \mathrm{NMR}$ $\left(50.3 \mathrm{MHz}, \mathrm{CDCl}_{3}\right): \delta_{\mathrm{c}}=19.2,26.8,31.6,36.1,50.0,67.6,79.9,209.7$. IR (neat): $\tilde{v}=3465$ (br, w), 2975 (m), 2874 (w), 2105 (s), 1703 (m), 1444 (w), 1408 (w), 1362 (m), 1306 (w), $1258(\mathrm{~m}), 1169(\mathrm{~m}), 1117(\mathrm{~m}) \mathrm{cm}^{-1} \cdot \mathrm{MS}\left(\mathrm{DCI}, \mathrm{NH}_{3}\right): 384\left(\left(2 \mathrm{M}+\mathrm{NH}_{4}\right)^{+}, 1\right), 218$ $\left(\left(\mathrm{M}+\mathrm{NH}_{3}+\mathrm{NH}_{4}\right)^{+}, 5\right), 201\left(\left(\mathrm{M}+\mathrm{NH}_{4}\right)^{+}, 100\right), 184\left((\mathrm{M}+\mathrm{H})^{+}, 16\right)$.

General procedure for the synthesis of 1-amino-2-(alkylidene)cyclopentanes 4a-w: To a anhydrous THF solution $(15 \mathrm{ml})$ of $\mathbf{3}$ was added $\mathrm{PPh}_{3}\left(1.2\right.$ equiv) at $20{ }^{\circ} \mathrm{C}$ and the reaction mixture was stirred for $24 \mathrm{~h}$ at $45{ }^{\circ} \mathrm{C}$. The solution was cooled to ambient temperature and water $(80 \mathrm{ml})$ was added. The organic and the aqueous layer were separated and the latter was extracted with $\mathrm{CH}_{2} \mathrm{Cl}_{2}(4 \times 50 \mathrm{ml})$. The combined organic layers were extracted with brine (1 x $80 \mathrm{ml})$, dried $\left(\mathrm{Na}_{2} \mathrm{SO}_{4}\right)$, filtered and the solvent of the filtrate was removed in vacuo. The residue was purified by chromatography (silica gel, ether/petroleum ether $=1: 2$ or ether/petroleum ether $=1: 40 \rightarrow 1: 3)$ to give compounds 4 .

$N$-(2-Methylenecyclopentyl)malonamic acid ethyl ester (4a): Treatment of 3a (0.39 g, 1.55 mmol) with $\mathrm{PPh}_{3}$ yielded, after purification by chromatography (silica gel, ether/petroleum ether $=1: 40 \rightarrow 1: 3), 4 \mathbf{a}$ as a yellow solid $(0.19 \mathrm{~g}, 58 \%) .{ }^{1} \mathrm{H}$ NMR $(300 \mathrm{MHz}$, $\left.\mathrm{CDCl}_{3}\right): \delta=1.25\left(\mathrm{t},{ }^{3} J=7 \mathrm{~Hz}, 3 \mathrm{H}\right), 1.38(\mathrm{~m}, 1 \mathrm{H}), 1.62(\mathrm{~m}, 1 \mathrm{H}), 1.73(\mathrm{~m}, 1 \mathrm{H}), 2.16(\mathrm{~m}, 1$ H), $2.37(\mathrm{~m}, 2 \mathrm{H}), 3.31(\mathrm{~s}, 2 \mathrm{H}), 4.17\left(\mathrm{q},{ }^{3} J=7 \mathrm{~Hz}, 2 \mathrm{H}\right), 4.62\left(\mathrm{dd},{ }^{3} J=7 \mathrm{~Hz},{ }^{3} J=3 \mathrm{~Hz}, 1 \mathrm{H}\right)$, 4.98 (m, $2 \mathrm{H}), 7.05$ (br, $1 \mathrm{H}) .{ }^{13} \mathrm{C} \mathrm{NMR}\left(50.3 \mathrm{MHz}, \mathrm{CDCl}_{3}\right): \delta=14.0,22.3,30.9,33.8,41.2$, 53.7, 61.5, 106.8, 152.0, 164.7, 169.57. IR (neat) $\tilde{v}=3286$ (br, m), 2960 (s), 1750 (s), 1650 (s), 1547 (s), 1465 (w), $1417(\mathrm{w}), 1369$ (m), $1182(\mathrm{~s}), 1033(\mathrm{~m}), 885$ (w) cm ${ }^{-1}$. MS (EI, 70 $\mathrm{eV}): m / z(\%)=211\left(\mathrm{M}^{+}, 65\right), 123(18), 97(56), 96(100), 81$ (28), 69 (29). The exact 
molecular mass $m / z=211.1208 \pm 2 \mathrm{ppm}\left[\mathrm{M}^{+}\right]$for $\mathrm{C}_{11} \mathrm{H}_{17} \mathrm{NO}_{3}$ was confirmed by HRMS (EI, $70 \mathrm{eV})$.

$N$-(2-Methylenecyclopentyl)malonamic acid methyl ester (4b): Treatment of $\mathbf{3 b}(0.12 \mathrm{~g}$, 0.49 mmol) with $\mathrm{PPh}_{3}$ yielded, after purification by chromatography (silica gel, ether/petroleum ether $=1: 40 \rightarrow 1: 3), \mathbf{4 b}$ as a yellow oil $(0.06 \mathrm{~g}, 61 \%) .{ }^{1} \mathrm{H}$ NMR $(250 \mathrm{MHz}$, $\left.\mathrm{CDCl}_{3}\right): \delta=1.38(\mathrm{~m}, 1 \mathrm{H}), 1.62(\mathrm{~m}, 1 \mathrm{H}), 1.73(\mathrm{~m}, 1 \mathrm{H}), 2.16(\mathrm{~m}, 1 \mathrm{H}), 2.37(\mathrm{~m}, 2 \mathrm{H}), 3.34$ (s, $2 \mathrm{H}), 3.70(\mathrm{~s}, 3 \mathrm{H}), 4.62\left(\mathrm{dd},{ }^{3} \mathrm{~J}=7 \mathrm{~Hz},{ }^{3} \mathrm{~J}=3 \mathrm{~Hz}, 1 \mathrm{H}\right), 4.96(\mathrm{~s}, 2 \mathrm{H}), 7.05$ (br, $\left.1 \mathrm{H}\right) .{ }^{13} \mathrm{C}$ $\operatorname{NMR}\left(50.3 \mathrm{MHz}, \mathrm{CDCl}_{3}\right): \delta=22.3,30.8,33.7,40.9,52.3,53.7,106.8,151.9,164.6,169.9$. IR (neat) $\tilde{v}=3420(\mathrm{w}), 3365(\mathrm{w}), 3268(\mathrm{~m}), 2958(\mathrm{w}), 2854(\mathrm{w}), 1750(\mathrm{~s}), 1650(\mathrm{~s}), 1555$ (m), $1438(w), 1336(w), 1288$ (w), 1219 (w), $1156(w), 1024(w), 882(w) \mathrm{cm}^{-1}$. MS (EI, 70 $\mathrm{eV}): m / z(\%)=197\left(\mathrm{M}^{+}, 65\right), 96(100), 95(21), 81(45), 69(32), 59(18), 41(41)$. The exact molecular mass $m / z=197.1052 \pm 2 \mathrm{ppm}\left[\mathrm{M}^{+}\right]$for $\mathrm{C}_{10} \mathrm{H}_{15} \mathrm{NO}_{3}$ was confirmed by HRMS (EI, $70 \mathrm{eV}$ ). Anal. calcd for $\mathrm{C}_{10} \mathrm{H}_{15} \mathrm{NO}_{3}$ (197.11): C 60.88, H 7.61, N 7.10; found $\mathrm{C} 60.61, \mathrm{H}$ 7.73, N 6.95.

$N$-(2-Methylenecyclopentyl)malonamic acid benzyl ester (4c): Treatment of $3 \mathrm{c}(0.17 \mathrm{~g}$, 0.54 mmol) with $\mathrm{PPh}_{3}$ yielded, after purification by chromatography (silica gel, ether/petroleum ether $=1: 2), \mathbf{4 c}$ as a yellow oil $(0.08 \mathrm{~g}, 52 \%) .{ }^{1} \mathrm{H} \mathrm{NMR}\left(300 \mathrm{MHz}, \mathrm{CDCl}_{3}\right): \delta$ $=1.37(\mathrm{~m}, 1 \mathrm{H}), 1.58(\mathrm{~m}, \mathrm{~A}$ of $\mathrm{AB}, 1 \mathrm{H}), 1.71(\mathrm{~m}, \mathrm{~B}$ of $\mathrm{AB}, 1 \mathrm{H}), 2.15(\mathrm{~m}, 1 \mathrm{H}), 2.36(\mathrm{~m}, 2$ H), 3.37 (s, $2 \mathrm{H}), 4.63$ (m, $1 \mathrm{H}), 4.93(\mathrm{~m}, 2 \mathrm{H}), 5.16$ (s, $2 \mathrm{H}), 6.96$ (br, $1 \mathrm{H}), 7.32$ (m, $5 \mathrm{H})$. ${ }^{13} \mathrm{C} \mathrm{NMR}\left(75.5 \mathrm{MHz}, \mathrm{CDCl}_{3}\right): \delta=22.3,30.9,33.7,41.3,53.7,67.2,106.9,128.1,128.3$, 128.6, 134.9, 151.8, 164.5, 169.2. IR (neat): $\tilde{v}=3296$ (br, m), 3069 (w), 3035 (w), 2960 (m), $2870(\mathrm{~m}), 1741(\mathrm{~s}), 1650$ (s), 1548 (m), 1499 (w), $1453(\mathrm{w}), 1413(\mathrm{w}), 1380(\mathrm{w}), 1330$ (w), 1263 (m), $1214(\mathrm{~m}), 1156$ (m), 1118 (w), 1078 (w), 1018 (w), $747(\mathrm{w}), 698$ (w) $\mathrm{cm}^{-1}$. 
MS (EI, $70 \mathrm{eV}): m / z(\%)=273\left(\mathrm{M}^{+}, 19\right), 255(5), 182(15), 139(9), 96(18), 91(100), 79(8)$.

The exact molecular mass $m / z=273.1365 \pm 2 \mathrm{ppm}\left[\mathrm{M}^{+}\right]$for $\mathrm{C}_{16} \mathrm{H}_{19} \mathrm{NO}_{3}$ was confirmed by HRMS (EI, $70 \mathrm{eV})$.

$N$-(2-Methylenecyclopentyl)malonamic acid 2-methoxyethyl ester (4d): Treatment of 3d (0.33 g, $1.16 \mathrm{mmol}$ ) with $\mathrm{PPh}_{3}$ yielded, after purification by chromatography (silica gel, ether/petroleum ether $=1: 2), \mathbf{4 d}$ as a yellow oil $(0.17 \mathrm{~g}, 61 \%)$; triphenylphosphine oxide could not be separated. ${ }^{1} \mathrm{H}$ NMR (300 $\left.\mathrm{MHz}, \mathrm{CDCl}_{3}\right): \delta=1.37(\mathrm{~m}, 1 \mathrm{H}), 1.57(\mathrm{~m}, 1 \mathrm{H}), 1.72$ (m, $1 \mathrm{H}), 2.14(\mathrm{~m}, 1 \mathrm{H}), 2.36(\mathrm{~m}, 2 \mathrm{H}), 3.36(\mathrm{~s}, 3 \mathrm{H}), 3.37$ (s, $2 \mathrm{H}), 3.68(\mathrm{~m}, 2 \mathrm{H}), 4.27$ (m, 2 $\mathrm{H}), 4.63(\mathrm{~m}, 1 \mathrm{H}), 4.95(\mathrm{~m}, 2 \mathrm{H}), 7.01(\mathrm{br}, 1 \mathrm{H}) .{ }^{13} \mathrm{C} \mathrm{NMR}\left(75.5 \mathrm{MHz}, \mathrm{CDCl}_{3}\right): \delta=22.3$, $30.9,33.741 .3,53.7,58.9,64.2,70.0,106.8,151.8,164.5,169.3$. IR (neat): $\tilde{v}=3434$ (br, w), $3287(w), 3076(w), 2957$ (w), 2895 (w), 1742 (s), 1651 (s), 1549 (m), 1439 (w), 1412 (w), $1373($ w), $1330(w), 1281(w), 1247(w), 1180(m), 1126(m), 1098(w), 1037(w), 884$ (w), $723(\mathrm{w}), 697(\mathrm{w}), 542(\mathrm{w}) \mathrm{cm}^{-1}$. MS (EI, $\left.70 \mathrm{eV}\right): \mathrm{m} / \mathrm{z}(\%)=241\left(\mathrm{M}^{+}, 47\right), 165(6), 137$ (4), 123 (24), 97 (60), 96 (100), 81 (29), 69 (28), 59 (34), 45 (13). The exact molecular mass $m / z=241.1314 \pm 2 \mathrm{ppm}\left[\mathrm{M}^{+}\right]$for $\mathrm{C}_{12} \mathrm{H}_{19} \mathrm{NO}_{4}$ was confirmed by HRMS (EI, $70 \mathrm{eV}$ ).

$N$-(2-Methylidenecyclopentyl)malonamic acid isobutyl ester (4e): Treatment of 3e $(0.42$ g, 1.49 mmol) with $\mathrm{PPh}_{3}$ yielded, after purification by chromatography (silica gel, ether/petroleum ether $=1: 2), \mathbf{4 e}$ as a yellow oil $(0.20 \mathrm{~g}, 55 \%)$. In addition, $4 \mathbf{e}^{\prime}(0.04 \mathrm{~g}, 11 \%)$ was isolated as a yellow oil. ${ }^{1} \mathrm{H} \mathrm{NMR}\left(300 \mathrm{MHz}, \mathrm{CDCl}_{3}\right): \delta=0.84\left(\mathrm{~d},{ }^{3} \mathrm{~J}=7 \mathrm{~Hz}, 6 \mathrm{~Hz}\right), 1.33$ (m, $1 \mathrm{H}), 1.54(\mathrm{~m}, 1 \mathrm{H}), 1.67(\mathrm{~m}, 1 \mathrm{H}), 1.86\left(\mathrm{~m},{ }^{3} J=7 \mathrm{~Hz}, 1 \mathrm{H}\right), 2.08$ (m, $\left.1 \mathrm{H}\right), 2.29$ (m, 2 H), $3.26(\mathrm{~s}, 2 \mathrm{H}), 3.83\left(\mathrm{~d},{ }^{3} \mathrm{~J}=7 \mathrm{~Hz}, 2 \mathrm{H}\right), 4.55(\mathrm{~m}, 1 \mathrm{H}), 4.88(\mathrm{~m}, 2 \mathrm{H}), 7.07$ (br, $\left.1 \mathrm{H}\right) .{ }^{13} \mathrm{C}$ $\operatorname{NMR}\left(75.5 \mathrm{MHz}, \mathrm{CDCl}_{3}\right): \delta=18.8,22.3,27.4,30.7,33.6,41.2,53.5(\mathrm{CH}), 71.3,106.7$, 151.7, 164.7, 169.2. IR (neat): $\widetilde{v}=3277(w), 2963(w), 1741(\mathrm{~m}), 1641(\mathrm{~s}), 1555$ (s), 1468 
(w), $1418(w), 1396(w), 1374(w), 1362$ (w), $1355(w), 1330(w), 1274(m), 1249(w), 1178$ (w), $1154(w), 1018(w), 966(w), 889(w), 619(w) \mathrm{cm}^{-1}$. MS (EI, $\left.70 \mathrm{eV}\right): \mathrm{m} / \mathrm{z}(\%)=239$ $\left(\mathrm{M}^{+}, 46\right), 183$ (16), $166(8), 123$ (21), 104 (16), 96 (100), 81 (33), 80 (25), 69 (21), 57 (17), 41 (23). The exact molecular mass $m / z=239.1521 \pm 2 \mathrm{ppm}\left[\mathrm{M}^{+}\right]$for $\mathrm{C}_{13} \mathrm{H}_{21} \mathrm{NO}_{3}$ was confirmed by HRMS (EI, $70 \mathrm{eV})$.

\section{2-(1-iso-Butyloxycarbonylmethylidene)-3a-hydroxyhexahydrocyclopenta $[b]$ pyrrole}

(4e' $).{ }^{1} \mathrm{H}$ NMR $\left(300 \mathrm{MHz}, \mathrm{CDCl}_{3}\right): \delta=0.98\left(\mathrm{~d},{ }^{3} \mathrm{~J}=6 \mathrm{~Hz}, 6 \mathrm{H}\right), 1.57-1.97(\mathrm{~m}, 7 \mathrm{H}), 2.90$ (m, $2 \mathrm{H}), 3.78\left(\mathrm{~d},{ }^{3} \mathrm{~J}=6 \mathrm{~Hz}, 2 \mathrm{H}\right), 3.94\left(\mathrm{~d},{ }^{3} \mathrm{~J}=5 \mathrm{~Hz}, 1 \mathrm{H}\right), 4.41(\mathrm{~s}, 1 \mathrm{H}), 7.75(\mathrm{br}, 1 \mathrm{H}) .{ }^{13} \mathrm{C}$ $\operatorname{NMR}\left(75.5 \mathrm{MHz}, \mathrm{CDCl}_{3}\right): \delta_{\mathrm{c}}=19.2,24.2,28.0,33.1,41.1,46.8,68.9,71.1,76.7,86.2$, 164.4, 170.9. IR (neat): $\tilde{v}=3369$ (br, m), 1961 (m), 2935 (m), 1729 (m), 1653 (s), 1596 (m), 1465 (m), 1448 (m), 1412 (m), $1380(\mathrm{~m}), 1320(\mathrm{w}), 1285(\mathrm{w}), 1260(\mathrm{w}), 1234(\mathrm{~m}), 1200$ (m), 1174 (m), 1146 (m), 1125 (m), 1098 (m), 1042 (w) cm cm $^{-1}$ MS (EI, $\left.70 \mathrm{eV}\right): 239$ (M+2 23), 184 (64), 166 (61), 139 (100), 122 (8), 120 (10), 96 (28), 81 (27). HRMS (EI, 70 eV): The exact molecular mass $m / z=239.1521 \pm 2 \mathrm{ppm}\left[\mathrm{M}^{+}\right]$for $\mathrm{C}_{13} \mathrm{H}_{21} \mathrm{NO}_{3}$ was confirmed by HRMS (EI, $70 \mathrm{eV})$.

$N$-(2-Ethylidenecyclopentyl)malonamic acid methyl ester (4f): Treatment of $3 f(0.11 \mathrm{~g}$, 0.41 mmol) with $\mathrm{PPh}_{3}$ yielded, after purification by chromatography (silica gel, ether/petroleum ether $=1: 40 \rightarrow 1: 3), \mathbf{4 f}$ as a yellow oil $(0.05 \mathrm{~g}, 57 \%, E / Z=2: 1) .{ }^{1} \mathrm{H} \mathrm{NMR}$ (250 MHz, $\mathrm{CDCl}_{3}$, major isomer): $\delta=1.64\left(\mathrm{~d},{ }^{3} \mathrm{~J}=7 \mathrm{~Hz}, 3 \mathrm{H}\right), 1.30-2.40(\mathrm{~m}, 6 \mathrm{H}), 3.35$ (s, $2 \mathrm{H}), 3.75(\mathrm{~s}, 3 \mathrm{H}), 4.60(\mathrm{~m}, 1 \mathrm{H}), 5.40(\mathrm{~m}, 1 \mathrm{H}), 6.98(\mathrm{br}, 1 \mathrm{H}) .{ }^{13} \mathrm{C}$ NMR $(50.3 \mathrm{MHz}$, $\mathrm{CDCl}_{3}$, major isomer): $\delta=14.5,22.4,27.5,34.0,41.0,52.3,53.9,117.0,142.7,164.6$, 160.0. IR (neat) $\tilde{v}=3292(\mathrm{w}), 2956(\mathrm{~s}), 2857(\mathrm{~m}), 1746(\mathrm{~s}), 1650(\mathrm{~s}), 1546(\mathrm{~m}), 1437(\mathrm{~m})$, 1326 (w), 1261 (m), 1203 (m), 1165 (m), 1119 (w), 1020 (w) $\mathrm{cm}^{-1}$. MS (EI, $\left.70 \mathrm{eV}\right): \mathrm{m} / z(\%)$ 
$=211\left(\mathrm{M}^{+}, 26\right), 110(76), 95(100), 79(57), 57(94), 41(85)$. The exact molecular mass $\mathrm{m} / \mathrm{z}$ $=211.1208 \pm 2 \mathrm{ppm}\left[\mathrm{M}^{+}\right]$for $\mathrm{C}_{11} \mathrm{H}_{17} \mathrm{NO}_{3}$ was confirmed by HRMS (EI, $\left.70 \mathrm{eV}\right)$.

$N$-(2-Propylidenecyclopentyl)malonamic acid ethyl ester (4g): Treatment of $\mathbf{3 g}(0.24 \mathrm{~g}$, 0.85 mmol) with $\mathrm{PPh}_{3}$ yielded, after purification by chromatography (silica gel, ether/petroleum ether $=1: 40 \rightarrow 1: 3), \mathbf{4 g}$ as an orange oil $(0.10 \mathrm{~g}, 47 \%, E / Z=3: 1) .{ }^{1} \mathrm{H} \mathrm{NMR}$ $\left(250 \mathrm{MHz}, \mathrm{CDCl}_{3}\right.$, major isomer): $\delta=0.92\left(\mathrm{t},{ }^{3} \mathrm{~J}=7 \mathrm{~Hz}, 3 \mathrm{H}\right), 1.30\left(\mathrm{t},{ }^{3} \mathrm{~J}=7 \mathrm{~Hz}, 3 \mathrm{H}\right), 1.20-$ $2.40(\mathrm{~m}, 8 \mathrm{H}), 3.30(\mathrm{~s}, 2 \mathrm{H}), 4.18\left(\mathrm{q},{ }^{3} J=7 \mathrm{~Hz}, 2 \mathrm{H}\right), 4.78(\mathrm{~m}, 1 \mathrm{H}), 5.42\left(\mathrm{dt},{ }^{3} J=7 \mathrm{~Hz},{ }^{3} J=\right.$

$1 \mathrm{~Hz}, 1 \mathrm{H}), 7.00$ (br, $1 \mathrm{H}) .{ }^{13} \mathrm{C} \mathrm{NMR}\left(50.3 \mathrm{MHz}, \mathrm{CDCl}_{3}\right.$, major isomer): $\delta=14.0,14.122 .4$, 23.8, 32.8, 35.1, 41.2, 50.3, 61.4, 128.2, 141.1, 163.7, 169.5. IR (neat) $\tilde{v}=3294(\mathrm{w}), 2963$ (s), 2873 (m), 1743 (s), 1648 (s), 1544 (m), 1462 (w), 1416 (w), 1370 (m), 1300 (m), 1181 (m), $1097(\mathrm{w}), 1032(\mathrm{~m}) \mathrm{cm}^{-1} . \mathrm{MS}(\mathrm{EI}, 70 \mathrm{eV}): \mathrm{m} / z(\%)=239\left(\mathrm{M}^{+}, 14\right), 210(17), 148(22)$, 132 (33), 124 (40), 109 (63), 96 (100). The exact molecular mass $m / z=239.1521 \pm 2 \mathrm{ppm}$ $\left[\mathrm{M}^{+}\right]$for $\mathrm{C}_{13} \mathrm{H}_{21} \mathrm{NO}_{3}$ was confirmed by HRMS (EI, $70 \mathrm{eV}$ ).

$N$-(2-Methoxymethylidenecyclopentyl)malonamic acid methyl ester (4h): Treatment of 3h $(0.17 \mathrm{~g}, 0.62 \mathrm{mmol})$ with $\mathrm{PPh}_{3}$ yielded, after purification by chromatography (silica gel, ether/petroleum ether $=1: 40 \rightarrow 1: 3), \mathbf{4 h}$ as a yellow oil $(0.08 \mathrm{~g}, 57 \%, E / Z=3: 1) .{ }^{1} \mathrm{H}$ NMR (250 MHz, $\mathrm{CDCl}_{3}$, major isomer): $\delta=1.40-2.40(\mathrm{~m}, 6 \mathrm{H}), 3.33(\mathrm{~s}, 2 \mathrm{H}), 3.56(\mathrm{~s}, 3 \mathrm{H}), 3.75$ $(\mathrm{s}, 3 \mathrm{H}), 4.70\left(\mathrm{dd},{ }^{3} \mathrm{~J}=7 \mathrm{~Hz},{ }^{3} \mathrm{~J}=3 \mathrm{~Hz}, 1 \mathrm{H}\right), 6.05$ (s, $\left.1 \mathrm{H}\right), 6.98(\mathrm{br}, 1 \mathrm{H}) .{ }^{13} \mathrm{C}$ NMR $(50.3$ $\mathrm{MHz}, \mathrm{CDCl}_{3}$, major isomer): $\delta=23.1,26.1,34.4,41.0,51.8,52.3,59.7,119.9,142.7,164.1$, 169.9. IR (neat): $\tilde{v}=3293$ (w), 2956 (m), $2851(\mathrm{w}), 1743$ (s), 1656 (s), $1549(\mathrm{~m}), 1438(\mathrm{~m})$, 1343 (w), 1280 (w), 1203 (m), 1167 (m), 1122 (w), 1019 (w) cm . $^{-1}$ MS (EI, 70 eV): m/z (\%) $=227\left(\mathrm{M}^{+}, 46\right), 195(52), 126(100), 110(88), 95(56), 45$ (58), 43 (45). The exact molecular mass $m / z=227.1158 \pm 2 \mathrm{ppm}\left[\mathrm{M}^{+}\right]$for $\mathrm{C}_{11} \mathrm{H}_{17} \mathrm{NO}_{4}$ was confirmed by HRMS (EI, $\left.70 \mathrm{eV}\right)$. 
$N$-(2-Methylidenecyclopentyl)-2-methylmalonamic acid ethyl ester (4i): Treatment of 3i $(0.12 \mathrm{~g}, 0.45 \mathrm{mmol})$ with $\mathrm{PPh}_{3}$ yielded, after purification by chromatography (silica gel, ether/petroleum ether $=1: 40 \rightarrow 1: 3), \mathbf{4 i}$ as a yellow oil $(0.06 \mathrm{~g}, 58 \%$, diastereomeric mixture $)$. ${ }^{1} \mathrm{H} \mathrm{NMR} \mathrm{(300} \mathrm{MHz,} \mathrm{CDCl}_{3}$, major isomer): $\delta=1.20\left(\mathrm{t},{ }^{3} J=7 \mathrm{~Hz}, 3 \mathrm{H}\right), 1.39\left(\mathrm{~d},{ }^{3} J=7 \mathrm{~Hz}, 3\right.$ H), $1.35(\mathrm{~m}, 1 \mathrm{H}), 1.54(\mathrm{~m}, 1 \mathrm{H}), 1.69(\mathrm{~m}, 1 \mathrm{H}), 2.12(\mathrm{~m}, 1 \mathrm{H}), 2.34(\mathrm{~m}, 2 \mathrm{H}), 3.25\left(\mathrm{q},{ }^{3} J=7\right.$ $\mathrm{Hz}, 1 \mathrm{H}), 4.16\left(\mathrm{q},{ }^{3} J=7 \mathrm{~Hz}, 2 \mathrm{H}\right), 4.58(\mathrm{~m}, 1 \mathrm{H}), 4.90\left(\mathrm{~d},{ }^{2} J=6 \mathrm{~Hz}, 2 \mathrm{H}\right), 6.42$ (br, $\left.1 \mathrm{H}\right) .{ }^{13} \mathrm{C}$ $\operatorname{NMR}\left(50.3 \mathrm{MHz}, \mathrm{CDCl}_{3}\right.$, major isomer): $\delta=14.0,14.9,22.2,30.8,33.7,46.8,53.6,61.4$ 106.6, 152.1, 168.7, 172.3. IR (neat): $\tilde{v}=3273$ (m), 2988 (w), 2939 (w), 1751 (s), 1645 (s), 1557 (m), 1452 (w), 1374 (w), 1326 (w), 1242 (m), 1200 (s), 1097 (w) cm ${ }^{-1}$. MS (EI, 70 eV): $m / z(\%)=225\left(\mathrm{M}^{+}, 6\right), 125(56), 96(64), 69(57), 55(33), 43$ (41), 41 (100). The exact molecular mass $m / z=225.1365 \pm 2 \mathrm{ppm}\left[\mathrm{M}^{+}\right]$for $\mathrm{C}_{12} \mathrm{H}_{19} \mathrm{NO}_{3}$ was confirmed by HRMS (EI, $70 \mathrm{eV})$.

2-Benzyl- $N$-(2-methylidenecyclopentyl)malonamic acid ethyl ester (4j): Treatment of $3 \mathbf{j}$ (0.17 $\mathrm{g}, 0.49 \mathrm{mmol})$ with $\mathrm{PPh}_{3}$ yielded, after purification by chromatography (silica gel, ether/petroleum ether $=1: 2), \mathbf{4 j}$ as a yellow oil $(0.07 \mathrm{~g}, 50 \%$, diastereomeric mixture $)$. In addition, 4j' (0.02 g, 17\%, diastereomeric mixture) was isolated as a yellow oil. ${ }^{1} \mathrm{H}$ NMR (300 MHz, $\mathrm{CDCl}_{3}$, major isomer): $\delta=1.15\left(\mathrm{t},{ }^{3} \mathrm{~J}=7 \mathrm{~Hz}, 3 \mathrm{H}\right), 1.20-2.44(\mathrm{~m}, 6 \mathrm{H}), 3.24$ (m, $1 \mathrm{H}), 3.47(\mathrm{~m}, 2 \mathrm{H}), 4.08\left(\mathrm{q},{ }^{3} J=7 \mathrm{~Hz}, 2 \mathrm{H}\right), 4.57$ (m, $\left.1 \mathrm{H}\right), 4.64-4.95$ (m, $\left.2 \mathrm{H}\right), 7.08$ (m, 5 H). ${ }^{13} \mathrm{C}$ NMR (75.5 MHz, $\mathrm{CDCl}_{3}$, major isomer): $\delta=14.0,22.3,30.9,33.8,36.5,53.7,54.9$, $61.4,106.8,126.7,128.4,128.9,137.9,152.0,167.4,171.2 . \mathrm{IR}$ (neat): $\tilde{v}=3200$ (br, m), 3086 (w), 3060 (w), 3031 (w), 2976 (s), 2932 (s), 2900 (s), 2864 (s), 1742 (s), 1654 (s), 1605 (m), 1585 (m), 1541 (m), 1495 (m), 1455 (m), 1445 (m), 1414 (m), 1382 (m), 1367 (m), 1350 (m), 1334 (m), 1283 (m), 1247 (m), 1181 (s), 1164 (s), 1153 (s), 1120 (s), 1079 (m), 
$1044(\mathrm{~m}), 1024(\mathrm{~m}), 749(\mathrm{~m}), 699(\mathrm{~m}) \mathrm{cm}^{-1}$. MS (EI, $\left.70 \mathrm{eV}\right): \mathrm{m} / z(\%)=301\left(\mathrm{M}^{+}, 45\right), 228$ (28), 210 (6), 192 (16), 177 (36), 159 (19), 148 (25), 131 (74), 97 (82), 91 (100), 81 (36), 69 (30), 41 (28). The exact molecular mass $m / z=301.1678 \pm 2 \mathrm{ppm}\left[\mathrm{M}^{+}\right]$for $\mathrm{C}_{18} \mathrm{H}_{23} \mathrm{NO}_{3}$ was confirmed by HRMS (EI, $70 \mathrm{eV})$.

\section{2-(1-Ethoxycarbonyl-2-phenyl-2-ethylidene)-3a-hydroxyhexahydrocyclopenta $[b]$ pyrrole}

$\left(\mathbf{4 j}^{\prime}\right) .{ }^{1} \mathrm{H}$ NMR $\left(300 \mathrm{MHz}, \mathrm{CDCl}_{3}\right): \delta=1.27\left(\mathrm{t},{ }^{3} \mathrm{~J}=7 \mathrm{~Hz}, 3 \mathrm{H}\right), 1.38-2.04(\mathrm{~m}, 6 \mathrm{H}), 2.83(\mathrm{~s}$, $2 \mathrm{H}), 3.47$ (s, $2 \mathrm{H}), 3.96\left(\mathrm{~d},{ }^{3} J=6 \mathrm{~Hz}, 1 \mathrm{H}\right), 4.08$ (q, $\left.{ }^{3} J=7 \mathrm{~Hz}, 2 \mathrm{H}\right), 7.18$ (m, $\left.5 \mathrm{H}\right), 8.19$ (br, $1 \mathrm{H}) .{ }^{13} \mathrm{C} \mathrm{NMR}\left(50.3 \mathrm{MHz}, \mathrm{CDCl}_{3}\right): \delta_{\mathrm{c}}=14.2,24.3,29.7,32.1,41.5,46.2,58.8,71.1,86.4$, $125.4,127.8,128.0,142.7,150.5,163.8,170.9$. IR (neat): $\tilde{v}=3416$ (br, w), 3337 (w), 3028 (w), 2974 (s), 2956 (s), 2928 (s), 2856 (s), 1734 (m), 1659 (m), 1586 (m), 1537 (w), 1494 (w), 1453 (m), 1415 (w), $1380(\mathrm{~m}), 1351(\mathrm{w}), 1261(\mathrm{~m}), 1240(\mathrm{~m}), 1201(\mathrm{~m}), 1181(\mathrm{~m}), 1119$ (s), 1076 (m), 1043 (m) cm ${ }^{-1}$. MS (EI, $\left.70 \mathrm{eV}\right): 301\left(\mathrm{M}^{+}, 76\right), 283$ (11), 272 (43), 254 (11), 228 (39), 210 (13), 192 (100), 177 (10), 146 (38), 125 (9), 111 (9), 97 (17), 91 (32), 83 (15), 69 (21), 57 (33), 43 (26). HRMS (EI, $70 \mathrm{eV}$ ): The exact molecular mass $\mathrm{m} / z=301.1678 \pm 2$ ppm $\left[\mathrm{M}^{+}\right]$for $\mathrm{C}_{18} \mathrm{H}_{23} \mathrm{NO}_{3}$ was confirmed by HRMS (EI, $70 \mathrm{eV}$ ).

2-Ethyl- $N$-(2-methylidenecyclopentyl)malonamic acid ethyl ester (4k): Treatment of 3k (0.20 g, $0.71 \mathrm{mmol})$ with $\mathrm{PPh}_{3}$ yielded, after purification by chromatography (silica gel, ether/petroleum ether $=1: 2), \mathbf{4 k}$ as a yellow oil $(0.05 \mathrm{~g}, 49 \%$, diastereomeric mixture $) .{ }^{1} \mathrm{H}$ NMR (300 MHz, $\mathrm{CDCl}_{3}$, major isomer): $\delta=1.95(\mathrm{~m}, 3 \mathrm{H}), 1.25\left(\mathrm{t},{ }^{3} \mathrm{~J}=7 \mathrm{~Hz}, 3 \mathrm{H}\right), 1.20-$ $2.43(\mathrm{~m}, 8 \mathrm{H}), 3.24(\mathrm{~m}, 1 \mathrm{H}), 4.17\left(\mathrm{q},{ }^{3} J=7 \mathrm{~Hz}, 2 \mathrm{H}\right), 4.61(\mathrm{~m}, 1 \mathrm{H}), 4.92(\mathrm{~m}, 2 \mathrm{H}), 6.54$ (br, $1 \mathrm{H}) .{ }^{13} \mathrm{C} \mathrm{NMR}\left(75.5 \mathrm{MHz}, \mathrm{CDCl}_{3}\right.$, major isomer): $\delta=11.7,14.1,22.3,24.6,30.9,33.8$, 53.5, 54.5, 61.3, 106.6, 152.9, 168.1, 172.2. IR (neat) $\tilde{v}=3307$ (br, w), $2974(\mathrm{~s}), 2936(\mathrm{~m})$, 2902 (m), 2874 (m), 1743 (s), 1650 (s), 1540 (m), 1460 (m), 1447 (m), 1389 (m), 1371 (m), 
1349 (w), 1260 (m), $1221(\mathrm{~m}), 1182$ (m), $1154(\mathrm{~m}), 1120(\mathrm{~m}), 1079(\mathrm{w}), 1026(\mathrm{w}) \mathrm{cm}^{-1} . \mathrm{MS}$ (EI, $70 \mathrm{eV}): m / z(\%)=239\left(\mathrm{M}^{+}, 16\right), 194(6), 143(9), 125$ (29), 97 (77), $81(25), 69$ (41), 55 (47), 41 (100). The exact molecular mass $m / z=239.1521 \pm 2 \mathrm{ppm}\left[\mathrm{M}^{+}\right]$for $\mathrm{C}_{13} \mathrm{H}_{21} \mathrm{NO}_{3}$ was confirmed by HRMS (EI, $70 \mathrm{eV})$.

2-n-Butyl- $N$-(2-methylidenecyclopentyl)malonamic acid ethyl ester (4I): Treatment of 3I (0.15 g, $0.48 \mathrm{mmol}$ ) with $\mathrm{PPh}_{3}$ yielded, after purification by chromatography (silica gel, ether/petroleum ether $=1: 2), \mathbf{4 l}$ as a yellow oil $(0.06 \mathrm{~g}, 51 \%$, diastereomeric mixture $)$. In addition, $\mathbf{4 l}^{\prime}$ was isolated $(0.02 \mathrm{~g}, 19 \%$, diastereomeric mixture $)$ as a yellow oil. ${ }^{1} \mathrm{H}$ NMR (300 MHz, $\mathrm{CDCl}_{3}$, major isomer): $\delta=1.94\left(\mathrm{t},{ }^{3} \mathrm{~J}=\mathrm{Hz}, 3 \mathrm{H}\right), 1.23\left(\mathrm{t},{ }^{3} \mathrm{~J}=7 \mathrm{~Hz}, 3 \mathrm{H}\right), 1.20-$ $2.44(\mathrm{~m}, 12 \mathrm{H}), 3.20(\mathrm{~m}, 1 \mathrm{H}), 4.17\left(\mathrm{q},{ }^{3} \mathrm{~J}=7 \mathrm{~Hz}, 2 \mathrm{H}\right), 4.61(\mathrm{~m}, 1 \mathrm{H}), 4.92(\mathrm{~m}, 2 \mathrm{H}), 6.48$ (br, $1 \mathrm{H}) .{ }^{13} \mathrm{C} \mathrm{NMR}\left(75.5 \mathrm{MHz}, \mathrm{CDCl}_{3}\right.$, major isomer). $\delta=13.7,14.1,22.3,27.4,27.7,29.4$, 30.9, 33.8, 53.5, 54.7, 61.3, 106.7, 152.2, 168.3, 172.4. IR (neat): $\tilde{v}=3367(\mathrm{w}), 3312(\mathrm{w})$, 2961 (s), 2932 (s), 2871 (s), 1739 (s), 1652 (m), 1538 (w), 1464 (m), 1448 (m), 1379 (m), $1349(\mathrm{w}), 1297(\mathrm{w}), 1228(\mathrm{w}), 1255(\mathrm{w}), 1220(\mathrm{w}), 1181(\mathrm{~m}), 1154(\mathrm{~m}), 1121(\mathrm{~m}), 1076(\mathrm{w})$,

$1025(\mathrm{w}) \mathrm{cm}^{-1}$. MS (EI, $\left.70 \mathrm{eV}\right): \mathrm{m} / z(\%)=267\left(\mathrm{M}^{+}, 5\right), 229(6), 187$ (8), $183(11), 171(10)$, 144 (8), 125 (37), 115 (9), 101 (27), 97 (100), 81 (22), 69 (43). The exact molecular mass $m / z=267.1834 \pm 2 \mathrm{ppm}\left[\mathrm{M}^{+}\right]$for $\mathrm{C}_{15} \mathrm{H}_{25} \mathrm{NO}_{3}$ was confirmed by HRMS (EI, $70 \mathrm{eV}$ ).

\section{2-(1-Ethoxycarbonyl-1-pentylidene)-3a-hydroxyhexahydrocyclopenta[b]pyrrole $\left(4 \mathrm{l}^{\prime}\right) .{ }^{1} \mathrm{H}$}

NMR (300 MHz, $\left.\mathrm{CDCl}_{3}\right): \delta=1.85(\mathrm{~m}, 3 \mathrm{H}), 1.25(\mathrm{~m}, 7 \mathrm{H}), 1.64(\mathrm{~m}, 2 \mathrm{H}), 1.78(\mathrm{~m}, 2 \mathrm{H})$, $1.93(\mathrm{~m}, 2 \mathrm{H}), 2.04\left(\mathrm{t},{ }^{3} J=6 \mathrm{~Hz}, 2 \mathrm{H}\right), 2.85(\mathrm{~s}, 2 \mathrm{H}), 3.93\left(\mathrm{~d},{ }^{3} J=5 \mathrm{~Hz}, 1 \mathrm{H}\right), 4.09\left(\mathrm{q},{ }^{3} J=7\right.$ $\mathrm{Hz}, 2 \mathrm{H}), 8.01$ (br, $1 \mathrm{H}) .{ }^{13} \mathrm{C} \mathrm{NMR}\left(75.5 \mathrm{MHz}, \mathrm{CDCl}_{3}\right): \delta_{\mathrm{c}}=14.1,146,22.5,24.3,27.3$, 29.7, 32.9, 41.4, 45.8, 58.6, 70.9, 86.4, 89.2, 161.8, 170.8. IR (neat): $\tilde{v}=3340$ (br, w), 2975 (s), 2929 (s), 2860 (s), 1731 (m), 1661 (m), 1591 (w), 1460 (m), 1448 (m), 1410 (m), 1380 
(m), 1350 (w), 1297 (w), 1254 (m), 1207 (m), 1121 (s), 1076 (w), 1042 (w), 1027 (w) cm c $^{-1}$ MS (EI, 70 eV): 267 (M+ 12), 234 (7), 224 (100), 178 (12), 139 (9), 132 (15), 97 (8), 69 (9), $57(13)$.

$\mathbf{N}$-(2-Methylidenecyclopentyl)acetamide (4m): Treatment of $\mathbf{3 m}(0.18 \mathrm{~g}, 0.80 \mathrm{mmol})$ with $\mathrm{PPh}_{3}$ yielded, after purification by chromatography (silica gel, ether/petroleum ether $=1: 2$ ), 4m as a yellow oil $(0.05 \mathrm{~g}, 37 \%)$; triphenylphosphine oxide could not be separated. ${ }^{1} \mathrm{H}$ NMR (300 MHz, $\left.\mathrm{CDCl}_{3}\right): \delta=1.38(\mathrm{~m}, 1 \mathrm{H}), 1.57(\mathrm{~m}, \mathrm{H}), 1.71(\mathrm{~m}, 1 \mathrm{H}), 2.11(\mathrm{~m}, 1 \mathrm{H}), 2.23(\mathrm{~s}$, $3 \mathrm{H}), 2.35(\mathrm{~m}, 2 \mathrm{H}), 3.41(\mathrm{~s}, 2 \mathrm{H}), 4.59(\mathrm{~m}, 1 \mathrm{H}), 4.94(\mathrm{~m}, 2 \mathrm{H}), 6.92$ (br, $1 \mathrm{H}) .{ }^{13} \mathrm{C} \mathrm{NMR}$ $\left(75.5 \mathrm{MHz}, \mathrm{CDCl}_{3}\right): \delta=22.3,30.8,30.9,33.749 .7,53.6,106.8,151.8,165.2,204.5 . \mathrm{IR}$ (neat): $\tilde{v}=3299$ (br, m), $3076(w), 2960$ (m), 2105 (w), 1719 (s), 1645 (s), 1549 (s), 1432 (m), 1415 (m), 1359 (m), 1323 (w), 1258 (w), $1216(\mathrm{w}), 1180(\mathrm{w}), 1159$ (m), $1119(\mathrm{w}), 1068$ (w), $1048(\mathrm{w}), 1030(\mathrm{w}), 1001(\mathrm{w}) \mathrm{cm}^{-1}$. MS (EI, $\left.70 \mathrm{eV}\right): \mathrm{m} / \mathrm{z}(\%)=181\left(\mathrm{M}^{+}, 51\right), 138(19)$, 123 (44), 96 (100), 82 (36), 69 (53). The exact molecular mass $m / z=181.1192 \pm 2 \mathrm{ppm}\left[\mathrm{M}^{+}\right]$ for $\mathrm{C}_{10} \mathrm{H}_{15} \mathrm{NO}_{2}$ was confirmed by HRMS (EI, $\left.70 \mathrm{eV}\right)$.

4,4-Dimethyl- $N$-(2-methylidenecyclopentyl)-3-oxopentanamide (4n): Treatment of 3n (0.04 g, 0.17 mmol) with $\mathrm{PPh}_{3}$ yielded, after purification by chromatography (silica gel, ether/petroleum ether $=1: 2), \mathbf{4 n}$ as a yellow oil $(0.01 \mathrm{~g}, 41 \%) .{ }^{1} \mathrm{H} \mathrm{NMR}\left(250 \mathrm{MHz}, \mathrm{CDCl}_{3}\right): \delta$ $=1.18(\mathrm{~s}, 9 \mathrm{H}), 1.34-2.46(\mathrm{~m}, 6 \mathrm{H}), 3.43\left(\mathrm{~d},{ }^{2} J=4 \mathrm{~Hz}, 2 \mathrm{H}\right), 4.65(\mathrm{~m}, 1 \mathrm{H}), 4.96(\mathrm{~m}, 2 \mathrm{H})$, 7.06 (br, $1 \mathrm{H}) .{ }^{13} \mathrm{C}$ NMR $\left(75.5 \mathrm{MHz}, \mathrm{CDCl}_{3}\right): \delta=22.3,25.9,29.7,30.9,33.8,43.4,53.7$, 106.8, 152.0, 165.6, 198.6. IR (neat): $\tilde{v}=3305$ (br, w), 2963 (s), 2924 (s), 2854 (s), 1711 (s), 1640 (s), 1548 (m), 1462 (m), 1408 (w), $1384(w), 1366$ (w), $1323(w), 1285$ (w), 1264 (w), 1219 (w), 1176 (w), 1156 (w), 1120 (m), 1065 (w), 1019 (w) $\mathrm{cm}^{-1}$. MS (EI, $\left.70 \mathrm{eV}\right): \mathrm{m} / \mathrm{z}$ $(\%)=223\left(\mathrm{M}^{+}, 85\right), 167(27), 138(16), 125$ (42), 123 (21), 97 (100), 96 (62), 81 (51), 69 
(18), 57 (54), 41 (26). The exact molecular mass $\mathrm{m} / z=223.1572 \pm 2 \mathrm{ppm}\left[\mathrm{M}^{+}\right]$for $\mathrm{C}_{13} \mathrm{H}_{21} \mathrm{NO}_{2}$ was confirmed by HRMS (EI, $70 \mathrm{eV}$ ).

$N$-(2-Ethylidenecyclopentyl)-3-oxopentanamide (4o): Treatment of 30 (0.06 g, 0.22 mmol) with $\mathrm{PPh}_{3}$ yielded, after purification by chromatography (silica gel, ether/petroleum ether = 1:2), 40 as a yellow oil $(0.02 \mathrm{~g}, 38 \%, E / Z=2: 1) .{ }^{1} \mathrm{H} \mathrm{NMR}\left(250 \mathrm{MHz}, \mathrm{CDCl}_{3}\right.$, major isomer): $\delta=1.07\left(\mathrm{t},{ }^{3} J=7 \mathrm{~Hz}, 3 \mathrm{H}\right), 1.38(\mathrm{~m}, 1 \mathrm{H}), 1.53-1.73(\mathrm{~m}, 4 \mathrm{H}), 1.76(\mathrm{~m}, 1 \mathrm{H}), 2.12(\mathrm{~m}, 1$ H), $2.25(\mathrm{~m}, 2 \mathrm{H}), 2.57\left(\mathrm{q},{ }^{3} \mathrm{~J}=7 \mathrm{~Hz}, 2 \mathrm{H}\right), 3.42(\mathrm{~s}, 2 \mathrm{H}), 4.59(\mathrm{~m}, 1 \mathrm{H}), 5.19(\mathrm{~m}, 1 \mathrm{H}), 6.86$ (br, $1 \mathrm{H}) .{ }^{13} \mathrm{C}$ NMR (50.3 MHz, $\mathrm{CDCl}_{3}$, major isomer): $\delta=7.4,14.5,22.4,27.5,34.1,37.1$, 48.8, 53.9, 117.0, 142.7, 165.1, 198.6. IR (neat): $\tilde{v}=3505$ (br, s), 3071 (w), 2972 (s), 2936 (s), 2878 (s), 2861 (s), 1719 (s), 1646 (s), 1548 (s), 1456 (m), 1413 (m), 1377 (m), 1355 (m), $1321(\mathrm{~m}), 1294$ (m), 1260 (m), 1178 (m), 1109 (m), 1075 (m), 1045 (m), $1022(\mathrm{w}), 964(\mathrm{w})$ $\mathrm{cm}^{-1} . \mathrm{MS}(\mathrm{EI}, 70 \mathrm{eV}): \mathrm{m} / \mathrm{z}(\%)=209\left(\mathrm{M}^{+}, 53\right), 152(10), 137(19), 116$ (20), 110 (82), 96 (100), 79 (47), 67 (24), 57 (42), 41 (33). The exact molecular mass $m / z=209.1416 \pm 2 \mathrm{ppm}$ $\left[\mathrm{M}^{+}\right]$for $\mathrm{C}_{12} \mathrm{H}_{19} \mathrm{NO}_{2}$ was confirmed by HRMS (EI, $\left.70 \mathrm{eV}\right)$.

$N$-(2-Methylidenecyclopentyl)-3-oxo-3-phenylpropionamide (4p): Treatment of 3p (0.06 g, $0.20 \mathrm{mmol}$ ) with $\mathrm{PPh}_{3}$ yielded, after purification by chromatography (silica gel, ether/petroleum ether $=1: 2), \mathbf{4 p}$ as a yellow oil $(0.02 \mathrm{~g}, 45 \%) .{ }^{1} \mathrm{H} \mathrm{NMR}\left(250 \mathrm{MHz}, \mathrm{CDCl}_{3}\right): \delta$ $=1.43(\mathrm{~m}, 1 \mathrm{H}), 1.64(\mathrm{~m}, 1 \mathrm{H}), 1.77(\mathrm{~m}, 1 \mathrm{H}), 2.21(\mathrm{~m}, 1 \mathrm{H}), 2.41(\mathrm{~m}, 2 \mathrm{H}), 4.00(\mathrm{~s}, 2 \mathrm{H})$, $4.66(\mathrm{~m}, 1 \mathrm{H}), 4.99(\mathrm{~m}, 2 \mathrm{H}), 7.08(\mathrm{br}, 1 \mathrm{H}), 7.41-7.66(\mathrm{~m}, 5 \mathrm{H}) .{ }^{13} \mathrm{C} \mathrm{NMR}(75.5 \mathrm{MHz}$, $\left.\mathrm{CDCl}_{3}\right): \delta=22.3,30.9,33.9,45.4,53.8,106.9,128.6,128.9,134.1,136.2,151.9,165.5$, 198.6. IR (neat): $\tilde{v}=3306$ (br, m), 2960 (m), 2926 (m), 2855 (w), 1689 (s), 1641 (s), 1600 (m), $1574(\mathrm{~m}), 1548(\mathrm{~m}), 1449(\mathrm{~m}), 1432$ (w), $1406(\mathrm{w}), 1326(\mathrm{~m}), 1280(\mathrm{~m}), 1263(\mathrm{~m}), 1212$ (m), $1180(\mathrm{w}), 1096(\mathrm{~m}), 1075(\mathrm{~m}), 1018(\mathrm{~m}) \mathrm{cm}^{-1}$. MS (EI, $\left.70 \mathrm{eV}\right): \mathrm{m} / z(\%)=243\left(\mathrm{M}^{+}, 8\right)$, 
226 (6), 198 (4), 164 (3), 147 (7), 123 (8), 105 (100), 96 (23), 81 (9), 77 (67), 69 (14). The exact molecular mass $m / z=243.1259 \pm 2 \mathrm{ppm}\left[\mathrm{M}^{+}\right]$for $\mathrm{C}_{15} \mathrm{H}_{17} \mathrm{NO}_{2}$ was confirmed by HRMS (EI, $70 \mathrm{eV})$.

$N$-(2-Methylidenecyclopentyl)-2-tosylacetamide (4q): Compound 3q (260 mg, 0.77 mmol) was dissolved in dry THF (10 ml) and triphenylphosphine $(244 \mathrm{mg}, 0.93 \mathrm{mmol})$ was added. The reaction mixture was stirred at $45{ }^{\circ} \mathrm{C}$ for $38 \mathrm{~h}$. Water $(50 \mathrm{ml})$ and $\mathrm{CH}_{2} \mathrm{Cl}_{2}(50 \mathrm{ml})$ were added and the aqueous layer was extracted with $\mathrm{CH}_{2} \mathrm{Cl}_{2}(3 \times 50 \mathrm{ml})$. The combined organic layers were washed with brine, dried $\left(\mathrm{Na}_{2} \mathrm{SO}_{4}\right)$, filtered, and the solvent of the filtrate was removed in vacuo. The residue was purified by chromatography (silica gel, petroleum ether/ethylacetate $=3 / 1)$ to give $\mathbf{4 q}$ as a yellow solid $(46 \mathrm{mg}, 20 \%) .{ }^{1} \mathrm{H}$ NMR $\left(\mathrm{CDCl}_{3}, 300\right.$ MHz): $\delta=1.37-1.52(\mathrm{~m}, 1 \mathrm{H}), 1.52-1.71(\mathrm{~m}, 1 \mathrm{H}), 1.71-1.85(\mathrm{~m}, 1 \mathrm{H}), 2.07-2.20(\mathrm{~m}, 1 \mathrm{H})$ 2.36-2.44 (m, $2 \mathrm{H}), 2.45(\mathrm{~s}, 3 \mathrm{H}), 4.02\left(\mathrm{~d},{ }^{2} \mathrm{~J}=1.1 \mathrm{~Hz}, 2 \mathrm{H}\right), 4.58(\mathrm{~m}, \mathrm{br}, 1 \mathrm{H}), 5.03(\mathrm{~m}, 2 \mathrm{H})$,

$6.67\left(\mathrm{~d}, \mathrm{br},{ }^{3} J=7.3 \mathrm{~Hz}, 1 \mathrm{H}\right), 7.37\left(\mathrm{~d},{ }^{3} J=8.2 \mathrm{~Hz}, 2 \mathrm{H}\right), 7.79\left(\mathrm{~d},{ }^{3} J=8.2 \mathrm{~Hz}, 2 \mathrm{H}\right) .{ }^{13} \mathrm{C}$ NMR $\left(\mathrm{CDCl}_{3}, 75 \mathrm{MHz}\right): \delta=21.7,22.4,30.9,33.6,54.1,62.0,107.5,128.1,130.0,135.2,145.6$, 151.3, 160.3. IR (KBr, $\left.\mathrm{cm}^{-1}\right): \widetilde{v}=3331(\mathrm{~s}), 2993(\mathrm{~m}), 2964(\mathrm{~m}), 2917(\mathrm{~m}), 1650(\mathrm{~s}), 1521$ (s), 1324 (s), 1299 (m), 1159 (s), 1087 (m), 814 (m), 522 (m). MS (EI, 70 eV): m/z (\%) = $294.0\left([\mathrm{M}+1]^{+}, 2\right), 293.0\left(\mathrm{M}^{+}, 20\right), 214.0(4), 155.1(6), 138.1(100), 96.1$ (30), $91.0(36)$ 32.1 (23), 28.1 (90). HRMS (EI, $70 \mathrm{eV}$ ): calcd. for $\mathrm{C}_{15} \mathrm{H}_{19} \mathrm{NO}_{3} \mathrm{~S}\left(\mathrm{M}^{+}\right)$293.10802, found 293.10758.

$N$-(1-Methylidene-indan-1-yl)malonamic acid ethyl ester (4r): Treatment of $3 \mathbf{r}(0.28 \mathrm{~g}$, 0.92 mmol) with $\mathrm{PPh}_{3}$ yielded, after purification by chromatography (silica gel, ether/petroleum ether $=1: 2), \mathbf{4 r}$ as a yellow oil $\left(0.08 \mathrm{~g}, 35 \%\right.$, unseparable mixture $\mathbf{4 r} / \mathbf{4} \mathbf{r}^{\prime}=$ 4:1). ${ }^{1} \mathrm{H}$ NMR $\left(250 \mathrm{MHz}, \mathrm{CDCl}_{3}\right): \delta=1.28\left(\mathrm{t},{ }^{3} J=7 \mathrm{~Hz}, 3 \mathrm{H}\right), 2.78\left(\mathrm{dd},{ }^{2} J=15 \mathrm{~Hz},{ }^{3} J=5\right.$ 
$\mathrm{Hz}, 1 \mathrm{H}), 3.27$ (s, $2 \mathrm{H}), 3.47\left(\mathrm{dd},{ }^{2} J=14 \mathrm{~Hz},{ }^{3} J=7 \mathrm{~Hz}, 1 \mathrm{H}\right), 4.19\left(\mathrm{q},{ }^{3} J=7 \mathrm{~Hz}, 2 \mathrm{H}\right), 5.11$ (m, $2 \mathrm{H}), 5.58(\mathrm{~m}, 1 \mathrm{H}), 7.17-7.54(\mathrm{~m}, 4 \mathrm{H}) .{ }^{13} \mathrm{C} \mathrm{NMR}\left(50.3 \mathrm{MHz}, \mathrm{CDCl}_{3}\right): \delta=14.0,38.8$, $41.2,52.8,61.6,104.9,120.9,125.30,127.1,129.2,138.8,142.8,150.5,165.0,169.4$. IR (neat): $\tilde{v}=3343$ (br, w), 3286 (br, w), 2979 (w), 2932 (w), 1738 (s), 1642 (s), 1604 (m), $1545(\mathrm{~m}), 1466$ (m), 1443 (m), $1414(\mathrm{~m}), 1370(\mathrm{~m}), 1320(\mathrm{~m}), 1282(\mathrm{~m}), 1240(\mathrm{~m}), 1181$ (m), $1152(\mathrm{~m}), 1115(\mathrm{~m}), 1095(\mathrm{~m}), 1034(\mathrm{~m}) \mathrm{cm}^{-1} . \mathrm{MS}(\mathrm{EI}, 70 \mathrm{eV}): \mathrm{m} / \mathrm{z}(\%)=259\left(\mathrm{M}^{+}, 15\right)$, 214 (3), 145 (5), 128 (100), 115 (5). The exact molecular mass $m / z=259.1208 \pm 2 \mathrm{ppm}\left[\mathrm{M}^{+}\right]$ for $\mathrm{C}_{15} \mathrm{H}_{17} \mathrm{NO}_{3}$ was confirmed by HRMS (EI, $70 \mathrm{eV}$ ).

$N$-(1-Methylidene-indan-2-yl)-3-oxobutyramide (4s): Treatment of 3s (0.22 g, $0.80 \mathrm{mmol})$ with $\mathrm{PPh}_{3}$ yielded, after purification by chromatography (silica gel, ether/petroleum ether = 1:2), 4s as a yellow oil $(0.09 \mathrm{~g}, 52 \%) .{ }^{1} \mathrm{H}$ NMR $\left(250 \mathrm{MHz}, \mathrm{CDCl}_{3}\right): \delta=2.29(\mathrm{~s}, 3 \mathrm{H}), 2.78$ $\left(\mathrm{dd},{ }^{2} J=15 \mathrm{~Hz},{ }^{3} J=5 \mathrm{~Hz}, 1 \mathrm{H}\right), 3.48\left(\mathrm{dd},{ }^{2} J=14 \mathrm{~Hz},{ }^{3} J=7 \mathrm{~Hz}, 1 \mathrm{H}\right), 3.50(\mathrm{~s}, 2 \mathrm{H}), 5.21(\mathrm{~m}$, $2 \mathrm{H}), 5.58(\mathrm{~m}, 1 \mathrm{H}), 7.24(\mathrm{~m}, 4 \mathrm{H}) .{ }^{13} \mathrm{C} \mathrm{NMR}\left(50.3 \mathrm{MHz}, \mathrm{CDCl}_{3}\right): \delta=31.1,38.8,49.5,52.8$, 106.0, 120.9, 125.3, 127.1, 129.2, 138.7, 142.7, 150.4, 165.5, 204.4. MS (EI, $70 \mathrm{eV}): \mathrm{m} / \mathrm{z}$ $(\%)=229\left(\mathrm{M}^{+}, 13\right), 171(4), 145(6), 128(100), 115(8), 85(2), 43(8)$. The exact molecular mass $m / z=229.1103 \pm 2 \mathrm{ppm}\left[\mathrm{M}^{+}\right]$for $\mathrm{C}_{14} \mathrm{H}_{15} \mathrm{NO}_{2}$ was confirmed by HRMS (EI, $\left.70 \mathrm{eV}\right)$.

4,4-Dimethyl- $N$-(1-methylidene-indan-2-yl)-3-oxopentanoic acid amide (4t): Treatment of 3t (0.07 g, $0.22 \mathrm{mmol}$ ) with $\mathrm{PPh}_{3}$ yielded, after purification by chromatography (silica gel, ether/petroleum ether $=1: 2), \mathbf{4 t}$ as a yellow oil $\left(0.03 \mathrm{~g}, 50 \%\right.$, unseparable mixture $\mathbf{4 t} / \mathbf{4} \mathbf{t}^{\prime}=$ 1:1). ${ }^{1} \mathrm{H}$ NMR $\left(250 \mathrm{MHz}, \mathrm{CDCl}_{3}\right): \delta=1.08(\mathrm{~s}, 9 \mathrm{H}), 2.89\left(\mathrm{dm},{ }^{2} J=15 \mathrm{~Hz}, 1 \mathrm{H}\right), 3.39\left(\mathrm{dd},{ }^{2} J\right.$ $\left.=14 \mathrm{~Hz},{ }^{3} J=7 \mathrm{~Hz}, 1 \mathrm{H}\right), 3.52(\mathrm{~s}, 2 \mathrm{H}), 5.16(\mathrm{~m}, 2 \mathrm{H}), 5.61(\mathrm{~m}, 1 \mathrm{H}), 6.93(\mathrm{br}, 1 \mathrm{H}), 7.18-$ $7.36(\mathrm{~m}, 4 \mathrm{H}) .{ }^{13} \mathrm{C} \mathrm{NMR}\left(75.5 \mathrm{MHz}, \mathrm{CDCl}_{3}\right): \delta=27.8,39.1,41.3,45.3,69.8,114.3,125.8$, 126.9, 127.8, 129.8, 138.4, 140.9, 158.5, 165.3, 204.8. IR (neat): $\tilde{v}=3307(\mathrm{~s}), 2964(\mathrm{~m})$, 
2928 (m), 1601 (s), 1521 (s), 1464 (m), 1425 (w), 1396 (w), 1362 (w), 1314 (w), 1293 (m), 1247 (w), 1198 (w), 1115 (w), 1092 (w), 773 (w), 751 (w), 718 (w) cm cm $^{-1}$ MS (EI, 70 eV): $m / z(\%)=271\left(\mathrm{M}^{+}, 9\right), 253(2), 214(100), 172(9), 145(6), 131(17), 103(6), 85(6), 57$ (13), 41 (7). The exact molecular mass $m / z=271.1573 \pm 2 \mathrm{ppm}\left[\mathrm{M}^{+}\right]$for $\mathrm{C}_{17} \mathrm{H}_{21} \mathrm{NO}_{2}$ was confirmed by HRMS (EI, $70 \mathrm{eV})$.

$N$-(1-Methylidene-indan-2-yl)-3-oxo-3-phenylpropionamide (4u): Treatment of $3 \mathbf{u}(0.04$ g, 0.13 mmol) with $\mathrm{PPh}_{3}$ yielded, after purification by chromatography (silica gel, ether/petroleum ether $=1: 2), \mathbf{4 u}$ as a brownish oil $(0.02 \mathrm{~g}, 46 \%) .{ }^{1} \mathrm{H}$ NMR $(250 \mathrm{MHz}$, $\left.\mathrm{CDCl}_{3}\right): \delta=2.82\left(\mathrm{dd},{ }^{2} J=15 \mathrm{~Hz},{ }^{3} J=5 \mathrm{~Hz}, 1 \mathrm{H}\right), 3.53\left(\mathrm{dd},{ }^{2} J=14 \mathrm{~Hz},{ }^{3} J=7 \mathrm{~Hz}, 1 \mathrm{H}\right), 4.04$ $(\mathrm{s}, 2 \mathrm{H}), 5.19(\mathrm{~m}, 2 \mathrm{H}), 5.59(\mathrm{~m}, 1 \mathrm{H}), 7.32-8.07(\mathrm{~m}, 9 \mathrm{H}, \mathrm{Ph}) .{ }^{13} \mathrm{C} \mathrm{NMR}(75.5 \mathrm{MHz}$, $\left.\mathrm{CDCl}_{3}\right): \delta=38.9,45.3,52.9,105.0,121.0,125.3,127.1,128.4,128.6,128.9,134.1,136.2$, 138.8, 142.8, 150.5, 165.5, 203.1. IR (neat): $\tilde{v}=3275$ (br, w), 3061 (w), 2955 (m), 2924 (s), 2853 (m), 1689 (s), 1642 (s), 1623 (s), 1604 (s), 1570 (m), 1524 (m), 1493 (m), 1452 (m), $1391(\mathrm{w}), 1362(\mathrm{w}), 1338(\mathrm{w}), 1280(\mathrm{~m}), 1210(\mathrm{w}), 1186(\mathrm{w}), 1161(\mathrm{w}), 1119(\mathrm{w}), 1095(\mathrm{w})$,

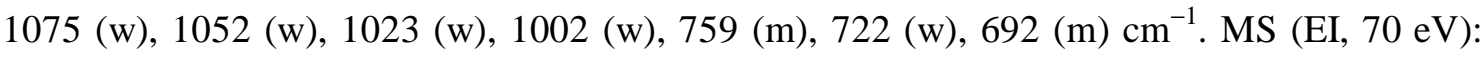
$m / z(\%)=291\left(\mathrm{M}^{+}, 64\right), 171(100), 145(82), 130(37), 105$ (79), 83 (24), 77 (75), 57 (50), 43 (41). The exact molecular mass $m / z=291.1259 \pm 2 \mathrm{ppm}\left[\mathrm{M}^{+}\right]$for $\mathrm{C}_{19} \mathrm{H}_{17} \mathrm{NO}_{2}$ was confirmed by HRMS (EI, $70 \mathrm{eV})$.

$N$-(2-Methylallyl)acetamide (4v): Treatment of 3v $(0.23 \mathrm{~g}, 1.15 \mathrm{mmol})$ with $\mathrm{PPh}_{3}$ yielded, after purification by chromatography (silica gel, ether/petroleum ether $=1: 2$ ), $\mathbf{4 v}$ as a yellow oil $(0.05 \mathrm{~g}, 30 \%) .{ }^{1} \mathrm{H}$ NMR (300 MHz, $\left.\mathrm{CDCl}_{3}\right): \delta=1.67$ (s, $\left.3 \mathrm{H}\right), 2.23$ (s, $\left.3 \mathrm{H}\right), 3.42(\mathrm{~s}, 2 \mathrm{H})$, $3.78\left(\mathrm{~d},{ }^{4} \mathrm{~J}=2 \mathrm{~Hz}, 2 \mathrm{H}\right), 4.79(\mathrm{~s}, 2 \mathrm{H}), 7.15$ (br, $\left.1 \mathrm{H}\right) \cdot{ }^{13} \mathrm{C} \mathrm{NMR}\left(75.5 \mathrm{MHz}, \mathrm{CDCl}_{3}\right): \delta=$ 20.3, 31.0, 44.9, 49.5, 110.9, 141.4, 165.3, 204.6. IR (neat): $\widetilde{v}=3310$ (br, s), 3083 (w), 
2975 (w), 2923 (w), 1717 (s), 1649 (s), 1551 (m), 1431 (m), 1361 (m), 1324 (w), 1163 (w), $1122(\mathrm{w}), 1095(\mathrm{w}), 1067(\mathrm{w}), 1025(\mathrm{w}) \mathrm{cm}^{-1}$. MS (EI, $\left.70 \mathrm{eV}\right): \mathrm{m} / z(\%)=155\left(\mathrm{M}^{+}, 51\right), 140$ (10), 112 (15), 97 (24), 85 (23), 71 (100), 70 (81). The exact molecular mass $\mathrm{m} / z=155.0946$ \pm 2 ppm $\left[\mathrm{M}^{+}\right]$for $\mathrm{C}_{8} \mathrm{H}_{13} \mathrm{NO}_{2}$ was confirmed by HRMS (EI, $\left.70 \mathrm{eV}\right)$.

$N$-(1,2-Dimethylallyl)-3-oxo-3-phenylpropionamide (4w): Treatment of 3w $(0.19 \mathrm{~g}, 0.70$ mmol) with $\mathrm{PPh}_{3}$ yielded, after purification by chromatography (silica gel, ether/petroleum ether $=1: 2), \mathbf{4 w}$ as a yellow oil $(0.10 \mathrm{~g}, 63 \%) .{ }^{1} \mathrm{H}$ NMR $\left(250 \mathrm{MHz}, \mathrm{CDCl}_{3}\right): \delta=1.28\left(\mathrm{~d},{ }^{3} J=\right.$ $7 \mathrm{~Hz}, 3 \mathrm{H}), 1.74(\mathrm{~s}, 3 \mathrm{H}), 3.96(\mathrm{~s}, 2 \mathrm{H}), 4.53(\mathrm{~m}, 1 \mathrm{H}), 4.86(\mathrm{~m}, 2 \mathrm{H}), 7.47$ (m, $5 \mathrm{H}) .{ }^{13} \mathrm{C} \mathrm{NMR}$ $\left(50.3 \mathrm{MHz}, \mathrm{CDCl}_{3}\right): \delta=19.6,38.4,45.3,49.9,110.4,128.5,128.8,134.1,136.1,145.7$, 164.7, 196.3. IR (neat): $\tilde{v}=3308$ (br, w), 2974 (w), 1692 (s), 1647 (s), 1599 (s), 1575 (s), 1548 (s), 1497 (w), 1449 (m), 1406 (w), 1377 (m), 1332 (m), 1283 (m), 1252 (w), 1229 (w), $1213(\mathrm{~m}), 1177(\mathrm{w}), 1162(\mathrm{w}) \mathrm{cm}^{-1} . \mathrm{MS}(\mathrm{EI}, 70 \mathrm{eV}): \mathrm{m} / \mathrm{z}(\%)=231\left(\mathrm{M}^{+}, 23\right), 214(34), 171$ (23), 147 (11), 126 (20), 105 (100), 84 (36), 77 (55), 70 (35). The exact molecular mass $\mathrm{m} / \mathrm{z}$ $=231.1259 \pm 2 \mathrm{ppm}\left[\mathrm{M}^{+}\right]$for $\mathrm{C}_{14} \mathrm{H}_{17} \mathrm{NO}_{2}$ was confirmed by HRMS (EI, $\left.70 \mathrm{eV}\right)$.

Ethyl 3a-hydroxyoctahydroindole-2-carboxylate (7): ${ }^{6}$ Treatment of $\mathbf{3 y}(0.58 \mathrm{~g}, 2.20$ mmol) with $\mathrm{PPh}_{3}$ yielded 7 as a greenish oil $(0.13 \mathrm{~g}, 27 \%) .{ }^{1} \mathrm{H}-\mathrm{NMR}\left(300 \mathrm{MHz}, \mathrm{CDCl}_{3}\right): \delta=$ $1.28\left(\mathrm{t},{ }^{3} J=7 \mathrm{~Hz}, 3 \mathrm{H}\right), 1.44-2.20(\mathrm{~m}, 8 \mathrm{H}), 2.45\left(\mathrm{~d},{ }^{2} J=15 \mathrm{~Hz}, 1 \mathrm{H}\right), 2.65\left(\mathrm{~d},{ }^{2} J=15 \mathrm{~Hz}, 1\right.$ $\mathrm{H}), 3.48\left(\mathrm{t},{ }^{3} \mathrm{~J}=6 \mathrm{~Hz}, 1 \mathrm{H}\right), 4.06,\left(\mathrm{q},{ }^{3} \mathrm{~J}=7 \mathrm{~Hz}, 2 \mathrm{H}\right), 4.54$ (s, $\left.1 \mathrm{H}\right), 7.23$ (br, $\left.1 \mathrm{H}\right) .{ }^{13} \mathrm{C}-\mathrm{NMR}$ $\left(50.3 \mathrm{MHz}, \mathrm{CDCl}_{3}\right): \delta=14.0,21.3,27.4,29.7,34.6,41.0,61.5,105.4,51.8,148.0,164.0$, 169.7. MS (EI, $70 \mathrm{eV}): m / z(\%)=225\left(\mathrm{M}^{+}, 73\right), 180(45), 162(21), 153(100), 134(41), 110$ (56). 
2-Methyl-1,4,5,6-tetrahydrocyclopenta[b]pyrrole (9): ${ }^{6}$ Starting with $\mathbf{3 y}(0.16 \mathrm{~g}, 0.90$ mmol) and $\mathrm{PPh}_{3}, 9$ was isolated $(0.11 \mathrm{~g}, 34 \%)$ as a brownish oil. ${ }^{1} \mathrm{H}$ NMR $(300 \mathrm{MHz}$, $\left.\mathrm{CDCl}_{3}\right): \delta=2.24(2,3 \mathrm{H}), 2.39\left(\mathrm{q},{ }^{3} J=5 \mathrm{~Hz}, 2 \mathrm{H}\right), 2.58\left(\mathrm{t},{ }^{3} J=5 \mathrm{~Hz}, 2 \mathrm{H}\right), 2.67\left(\mathrm{t},{ }^{3} J=5 \mathrm{~Hz}\right.$, $2 \mathrm{H}), 5.68$ (s, $1 \mathrm{H})$. The spectroscopic data were identical with those reported. ${ }^{6}$

\section{References}

1. Effenberger, F.; Beisswenger, T.; Az, R. Chem. Ber. 1985, 118, 4869.

2. Patonay, T.; Hoffman, R. V. J. Org. Chem. 1994, 59, 2902.

3. Forster, M. O.; Fierz, M. E. J. Chem. Soc. 1908, 93, 81.

4. Forster, M. O.; Fierz, M. E. J. Chem. Soc. 1908, 93, 675.

5. Batanero, B.; Escudero, J.; Barba, F. Synthesis 1999, 10, 1809.

6. Freifeld, I.; Shojaei, H.; Langer, P. J. Org. Chem. 2006, in press. 
